WEISS. - PROPRIÉTÉS MAGNÉTIQUES DE LA PYRRHOTINE 469

\title{
LES PROPRIÉTÉS MAGNÉTIQUES DE LA PYRRHOTINE;
}

Par M. Pierre WEISS ( $\left.{ }^{1}\right)$.

INTRODUCTION.

On ne rencontre guère, parmi les substances ferromagnétiques, elles-mêmes peu nombreuses, que deux corps bien cristallisés, la magnétite et la pyrrhotine. Une étude faite il y a quelques années $\left(^{2}\right)$ sur la magnétite m'a fait entrevoir toute la nouveauté des aperçus que l'on pouvait tirer de la considération simultanée des phénomènes magnétiques et de la structure cristalline. La plupart des recherches physiques sur les métaux magnétiques ont été faites, en effet, en ne considérant dans la matière qu'une seule direction privilégiée qui est, ou est censée être, à la fois celle de l'aimantation et celle du champ. Et les observations faites sous l'empire de cette conception linéaire du magnétisme ont été cataloguées dans un nombre considérable de courbes, auxquelles on ne rattache, en général, aucune tentative d'analyse des phénomènes.

Pour aborder le problème à trois dimensions qu'est réellement le magnétisme, il faut s’adresser non à des matières possédant la fausse isotropie des métaux usuels, à structure grenue, fibreuse ou lamellaire, formés le plus souvent de cristaux microscopiques enchevêtrés et ne pouvant donner que des moyennes indéchiffrables de phénomènes magnéto-cristallins, mais à des cristaux homogènes dans toute leur étendue, pour revenir, s'il est possible, des résultats plus simples qu'ils auront donnés aux propriètés des matières usuelles complexes.

A ce point de vue, l'étude de la pyrrhotine promettait, dès les premières expériences qui ont fait découvrir son plan magnétique, d'être particulièrement féconde. Le présent mémoire est consacré à l'exposé et la discussion de l'ensemble des résultats acquis sur cette substance.

(1) Conférence faite à la Société françaisc de Physique, le 28 avril 1903.

$\left.{ }^{2}\right)$ P. Werss, Ecl. électr., t. VII, p. 487; t. VIII, p. 56, 105: 1896: - J. de Plyys.; $3^{\circ}$ sérıe, t. V, p. 43 ว ; 1896.

J. de Phys., $4^{\mathrm{e}}$ série, t. IV. (Juillet 1905.) 


\section{CHAPITRE I ( $\left.{ }^{1}\right)$.}

\section{A. - Description de la pyrrhotine.}

La pyrrhotine est un sulfure de fer de composition voisine de celle du protosulfure $\mathrm{FeS}$, avec un excédent de soufre jusqu'à présent inexpliqué et qui n'est peut-être pas absolument constant. Citons deux analyses qui se rapportent à des substances de même origine que celles qui seront étudiées :

\begin{tabular}{lcc} 
& \multicolumn{2}{c}{ Composition } \\
& centésimale \\
Protosulfure FeS $\ldots \ldots \ldots \ldots \ldots \ldots \ldots$. & 36,39 & 63,61 \\
Pyrrhotine de Bodenmais (Rammelsberg).. & 38,83 & 61,17 \\
Pyrrhotine du Brésil (Berthier)........... & 37,38 & 62,62
\end{tabular}

La pyrrhotine contient fréquemment un peu de nickel.

On a proposé les formules $\mathrm{Fe}^{7} \mathrm{~S}^{8}, \mathrm{Fe}^{8} \mathrm{~S}^{9}, \mathrm{Fe}^{11} \mathrm{~S}^{12}$, qui toutes peuvent rentrer dans les types :

$$
n \mathrm{FeS}+\mathrm{Fe}^{2} \mathrm{~S}^{3} \quad \text { ou } \quad n \mathrm{FeS}+\mathrm{FeS}^{2} .
$$

La couleur varie du brun mat au bronze foncé.

La forme cristalline est considérée comme hexagonale par Romé de L'Isle et G. Rose. Ce dernier indique le rapport $a: e=\mathbf{1 , \boldsymbol { i } 4 0 2 .}$ On rencontre fréquemment la base du prisme $e(0001)$, ses faces latérales $m(10 \overline{1} 0)$ et un certain nombre de pyramides.

La structure de la matière est souvent feuilletée suivant $e$, G. Rose $\left(^{2}\right)$ remarque que ce n'est pas un clivage proprement dit.

J'ai trouvé pour la densité des substances que j'ai étudiées :

$$
\begin{array}{ccc}
\text { Pyrrhotine de Morro Velho. } & d=4,52 ; 4,58 ; 4,60 ; 4,605 . \\
\text { - } & \text { de Bodenmais.. } & 4,41-4,43 \\
- & \text { de New-Jersey.. } & \mathbf{4 , 5 6 - 4 , 5 9}
\end{array}
$$

Les deux dernières substances sont feuilletées; on est donc exposé à trouver leur densité trop faible. La densité exacte, si tant est qu'elle est définie, doit être voisine de 4,60.

Streng $\left({ }^{3}\right)$, par comparaison avec la Sternbergite, émet l'hypothèse

(1) Une grande partie des renseignements de ce chäpitre sont empruntés à Hintze, Handbuch der Mineralogie, t. I, p. 627.

(2) Pogg. Ann., 74, $296 ; 1849$.

(3) N. Jahrbuch für Mineral., p. 797; 1878. 
PROPRIÉTÉS MAGNÉTIQUES DE LA PYRRHOTINE 471 que la pyrrhotine pourrait être orthorhombique et doive son aspect hexagonal à un groupement de trois cristaux. Urba (1) confirme cette hypothèse par des mesures d'angles sur un cristal du Brésil. En 1882, Streng (2) revient de son opinion en concluant que, si les mesures d'angles ne donnent pas d'indications certaines, le clivage, les figures de corrosion et la conductibilité thermique indiquent nettement la forme hexagonale, " à laquelle les propriétés magnétiques ne contredisent pas $)$.

On obtient parl'acide chlorhydrique chaud des figures de corrosion dont les contours, absolument hexagonaux, sont fournis par desfaces de pyramides du premier ordre.

Fizeau a trouvé pour le coefficient de dilatation parallèle à l'axe $2,35 \times 10^{-6}$, et pour le coefficient de dilatation perpendiculaire $\dot{a}$ l'axe $31,20 \times 10^{-6}$.

Les meilleurs cristaux pour les études magnétiques proviennent de Morro Velho, au Brésil ; ils sont souvent d'apparence parfaitement homogène dans une étendue de plusieurs centimètres. Ils ne sont pas clivables; leur cassure conchoïdale a l'aspect d'un beau bronze foncé.

Par rapport aux propriétés magnétiques, douées d'une grande régularité, de cette première substance, que j'appellerai normale, les masses feuilletées provenant de Morro Velho, de New-Jersey et de Bodenmais présentent de nombreuses anomalies qui ont été l'objet d'une étude spéciale.

Enfin j'ai eu à ma disposition de petits cristaux de Schneeberg, dans le Tyrol, et des masses schisteuses de Norvège sur lesquelles je n'ai fait que peu de mesures; leur examen ne semblait pas devoir ajouter aux résultats donnés par les autres substances.

\section{B. - Expériences magnétiques antérieures.}

Les pyrrhotines de diverses provenances sont inégalement attirables à l'aimant, on en rencontre qui ne sont pas magnétiques du tout.

Elles sont rarement magnétipolaires; cependant, sur un échan-

(1) Groth's Ztschr., III, p. 190.

(2) N. Jahrb. für Mineral., p. $183 ; 1882,1$. 
tillon compact de Borév, Abt(1) a observé un champ coercitif voisin de 200 unités.

Streng $\left({ }^{2}\right)$ a fait sur la pyrrhotine des expériences magnétiques intéręssantes qui contiennent en germe la découverte du plan magnétique, mais sont restées incomplètes parce qu'elles sont purement qualitatives et que le seul critérium de l'aimantation mis en œuvre est le magnétisme rémanent. Je résume brièvement ces expériences d'après Hintze (loc. cit.). Des tables parallèles à la base possédant primitivement des pôles faibles deviennent nettement magnétipolaires quand elles sont frottées avec un aimant et se comportent alors comme l'acier, en ce sens que le point où la touche commence prend un pôle de même nom, et celui où elle s'arrête un pôle de nom contraire à celui de l'aimant frottant. Par contre, unc face taillée parallèlement à l'axe principal du cristal prend sur toute son étendue une polarité contraire à celle du pôle frottant la face opposée de la plaque, parallèle à la première, prenant du magnétisme de même nom que celui de ce pôle.

Streng ayant fixé un cube taillé suivant (0001) (1010) (11 20$)$ entre les pôles d'un électro-aimant puissant, l'axe de la pyrrhotine étant parallèle à l'axe de l'aimant, tenta ensuite d'examiner la polarité acquise en suspendant le cube dans une petite corbeille en papier, entre les pôles de l'aimant, dans la même position que dans l'opération précédente. Néanmoins, à la fermeture du courant, la substance se plaça toujours équatorialement, la direction perpendiculaire à l'axe cristallographique située dans la direction du champ devenant magnétipolaire.

Des résultats analogues furent obtenus avec un prisme de section carrée dont la longueur était parallèle à l'axe cristallographique. Après un essai d'aimantation dans le sens longitudinal, il était projeté violemment dans la position transversale. De même une plaque hexagonale parallèle à la base s'orienta toujours dans l'électro-aimant, l'axe se plaçant équatorialement.

Cette plaque suspendue, l'axe principal vertical, avait perdu sa faculté d'orientation en cc sens que, tournée d'un angle quelconque, elle resta dans la nouvelle position dans laquelle elle avait été maintenue un instant.

(1) Aвт, Wied. Ann., LVII, p. 135; 1896;-DU BoIs, Rapports du Congrès inlernational de Physique, t. II, p. 481 .

() Strexg, N. Jahrbuch für Mineralogie, p. 183;1882, 1;- Irstze. Handbuch der Mineralogie, 1. 1. p. 62 T. 
PROPRIÉTÉS MAGNÉTIQUES DE LA PYRRHOTINE 473

Un cylindre bas, obtenu en arrondissant cette plaque, montre aussi l'équivalence de toutes les directions perpendiculaires à l'axe principal et ne manifeste aucune polarité dans le sens de cet axe.

Toutes ces expériences en partie étranges s'expliquent aisément par les résultats du présent travail, qui les confirme, sauf en ce qui concerne l'isotropie magnétique dans le plan de base du prisme. Nous verrons que la symétrie de la substance est tout au plus orthorhombique.

\section{CHAPITRE Il.}

APPAREILS ET MÉthodes D'ObSERVATION.

1

\section{A. - Expériences d'induction.}

Les premières expériences ont été faites avec le galvanomètre balistique. La substance $p$ (fig. 1, 2 et 3 ), sous forme d'une sphère et plus tard d'un disque, se trouvait entre les pôles d'un aimant NS. Elle était fixée à la plate-forme d'un goniomètre, munie d'une alidade, tandis que les bobines induites $b, b^{\prime}$ étaient portées, au moyen d'un système de pièces de laiton, par l'alidade qui, en temps ordinaire, sert à la lecture des angles de la Junette. Ce système comprend les ressorts $\mathrm{R}$, qui permettent d'écarter les bobines, par le jeu des écrous $\mathrm{E}$, pour enlever la substance $p$ avec son support, et de les ramener ensuite exactement à lleur place. Dans la fig. 2, ce mouvement est indiqué par des tlèches. Les bobines avaient chacune 200 tours de fil fin de $0^{\mathrm{mm}}, 1$; elles entouraient la substance aussi exactement que possible et avaient à peu près la disposition des bobines d'une boussole de Helmholtz. Des butoirs tels que $e$ limitaient les angles de rotation de la substance ou des bobines.

Le champ magnétique était obtenu au moyen d'un aimant permanent d'abord, d'électro-aimants ensuite. Ces appareils étaient suspendus à un pied à crémaillère, semblable à ceux des appareils photographiques de grande dimension; ils pouvaient à volonté être rapprochés ou éloignés des bobines. Ce déplacement servait, avec l'aimant permanent, à faire varier l'intensité du champ; mais bientôt il a été évident que la partie la plus intéressante des phéno- 
mènes se rencontrerait dans les champs très intenses. L'aimantpermanent a été remplacé alors successivement par trois électroaimants, dont le plus puissant a permis d'atteindre des champs de 10000 gauss.

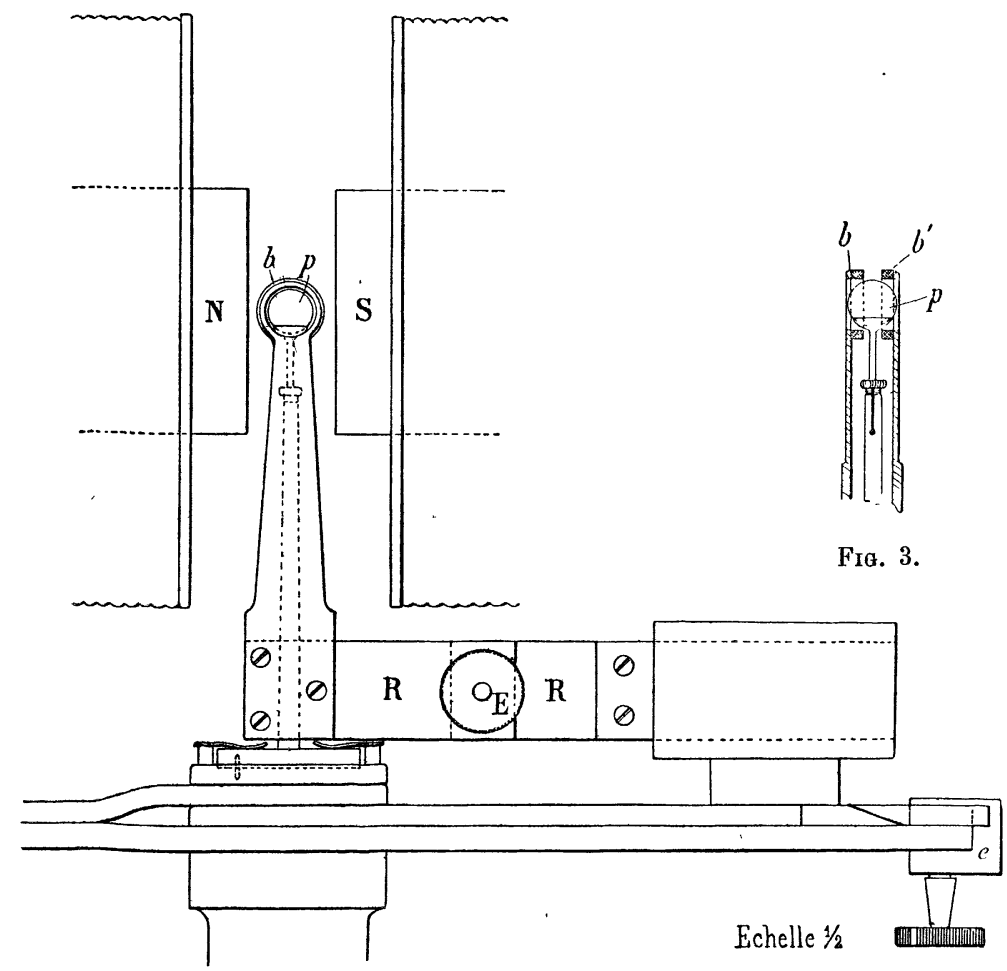

Fig. 1.

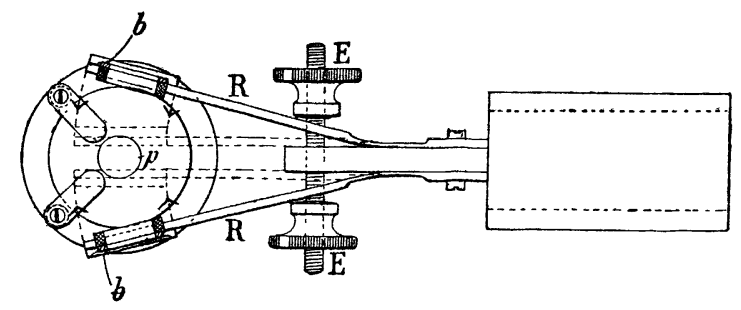

FIG. 2.

A plusieurs reprises, j'ai soupçonné le défaut d'uniformité du champ des électro-aimants d'être la cause de certaines irrégularités 
PROPRIÉTÉS MAGNÉTIQUES DE LA PYRRHOTINE 4T̃ dans les mesures; j'ai donc, à chaque reconstruction des appareils, pris des précautions plus grandes pour l'uniformité du champ. Les résultats n'en ont pas été modifiés; l'uniformité était donc suffisante.

Le problème expérimental, dans sa généralité, consiste à déterminer les trois composantes de l'intensité d'aimantation en fonction des trois composantes du champ. J'ai montré ailleurs (') comment il se simplifie quand on tient compte de la conservation du travail dans les transformations isothermiques réversibles. Dans ces expériences, je ne me suis appuyé sur cette propriété du travail que pour obtenir des vérifications (Voir loc. cit.) et j'ai abordé le problème directement, en mesurant successivement la composante de l'aimantation parallèle au champ et la composante horizontale perpendiculaire au champ, évitant seulement de rendre nécessaire la mesure de la composante verticale.

Calcul desobservations. - Soit un courant $i$ dans un circuit fixe ( $f g .4$ ), produisant, en un point quelconque A de l'espace, un champ exprimé par le vecteur $\mathrm{G} \times i$. G est la quantité dont l'emploi s'imposerait si l'on faisait du circuit électrique un galvanomètre. Je l'appellerai constante galvanométrique au point A.
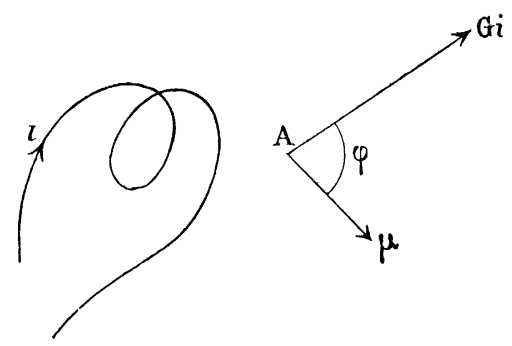

FIG. 4.

Supposons, pour la démonstration à faire, le courant $i$ constant, c'est-à-dire la force électromotrice de la source d'électricité constamment égale à la somme de celle qu'exige la loi d'Ohm et de celle qui surmonte la force électromotrice d'induction $\mathrm{E}$.

Produisons en A, par un procédé quelconque, un moment magnétique $\mu$ faisant avec $\mathrm{G} i$ un angle $\varphi$. L'énergie relative Gi $\mu \cos \varphi$

(1) C.R., 4 janvier $1904 ;-J$. de Phys., $4^{\circ}$ série, III, $194 ; 1904$. 
de $\mu$ et de l'appareil producteur du champ est transmise par le mecanisme de l'induction à la source. Elle est $\int \mathrm{E} i d t$. Donc

$$
\text { Gip. } \cos \varphi=\int \mathrm{E} d t
$$

Revenant maintenant au cas où le même circuit de résistance totale R, sans courant, contient un galvanomètre balistique, nous mesurerons, au moment de la production du moment $\mu$., une quantité d'électricité :

$$
q=\frac{1}{\mathrm{R}} \int \mathrm{E} d t=\frac{\mathrm{G}}{\mathrm{R}} \mu \cdot \cos \varphi, \cdot
$$

et, si nous étendons l'aimantation à un solide dont l'élément de volume est $d v$, en posant $\mu=\mathrm{I} d v$ :

$$
q=\frac{1}{\mathrm{R}} \int \mathrm{I}, \mathrm{G} \cos \varphi \mathrm{d} v
$$

Considérons deux cas particuliers :

$1^{\circ}$ Le circuit est quelconque, I est constant en grandeur et en direction dans toute l'étendue de la substance. Soient $\mathrm{I}_{x}, \mathrm{I}_{y}, \mathrm{I}_{z}, \mathrm{G}_{x}, \mathrm{G}_{y}, \mathrm{G}_{z}$, les composantes rectangulaires de 1 et de $\mathrm{G}$, on aura :

$$
q=\frac{1}{\mathrm{R}}\left(\mathrm{I}_{x} \int \mathrm{G}_{x} d v+\mathrm{I}_{y} \int \mathrm{G}_{y} d v+\mathrm{I}_{z} \int \mathrm{G}_{z} d v\right) .
$$

Si la substance est une sphère centrée par rapport aux bobines induites comme dans la fig. 3, et si l'axe des bobines est l'axe $\operatorname{des} x, \int \mathrm{G}_{y} d v$ et $\int \mathrm{G}_{z} d v$ seront nuls, et il restera:

$$
q=\frac{\mathrm{I}_{x}}{\mathrm{R}} \int \mathrm{G}_{x} d v
$$

Done, dans ce cas, le galvanomètre balistique mesurera la composante de l'aimantation parallèle à l'axe des bobines.

$2^{\circ}$ Le champ que produirait un courant $i$ dans les bobines induites est unifórme dans toute l'étendue de l'espace occupé par la substance, alors

$$
q=\frac{\mathrm{G}}{\mathrm{R}} \int \mathrm{I} \cos \varphi d v
$$


PROPRIÉTÉS MAGNÉTIQUES DE LA PYRRHOTINE 4Ti

et le galvanomètre balistique mesure exactement la valeur moyenne de la composante de l'aimantation dans la direction de ce champ uniforme.

Les conditions de l'expérience réalisent approximativement l'un et l'autre de ces cas particuliers, le premier lorsque le champ de l'aimant est uniforme, le second lorsque, comme dans la boussole de Helmholtz, les bobines ont l'écartement qui donne le champ le plus uniforme.

Par la rotation de la substance, placée dans un champ magnétique et entre les bobines induites immobiles, on obtiendra donc les variations de l'une ou de l'autre des deux composantes de l'aimantation, suivant que l'axe des bobines sera parallèle ou perpendiculaire au champ.

Mais il faut déterminer en outre, pour une position particulière de la substance, l'aimantation origine à partir de laquelle ces variations sont comptées.

Des raisons de symétrie donnent souvent les azimuts de la substance pour lesquels l'aimantation perpendiculaire au champ est nulle. On peut aussi les déterminer en cherchant par tâtonnements les positions de la substance où des variations d'intensité du champ n'agissent pas sur le galvanomètre. On s'est servi, avant la mise en place de la substance, du même critérium pour déterminer la position des bobines pour laquelle leur axe est perpendiculaire au champ.

Pour trouver la grandeur de la composante de l'aimantation parallèle au champ, on fait tourner les bobines induites de $180^{\circ}$, à partir de l'une des positions où leur axe est parallèle au champ, d'abord la substance étant en place, puis la substance enlevée. On voit immédiatement que la différence des deux indications du galvanomètre balistique donne l'intensité d'aimantation parallèle au champ avec la même unité avec laquelle ont été mesurées les variations par la rotation de la substance.

Valeur absolue. - Pour ramener ces mesures à des valeurs absolues, j'ai employé deux procédés.

On sait qu'une sphère de fer doux dans un champ magnétique $\mathrm{H}$, qui n'est passulfisant pour l'aimanter au delà de la région de forte perméabilité, prend une intensité d'aimantation donnée très approximativement par $\frac{3 H}{4 \pi}$. Elle est donc connue quand $H$ est connu. Opé- 
rant avec cette sphère comme avec la substance, on étalonne l'appareil pour les mesures d'intensité d'aimantation. Cette méthode donne de très bons résultats. Je lui ai préférć, dans la suite, celle qui consiste à mettre à la place de la substance une petite bobine d'aire connue, parcourue par un courant connu, c'est-à-dire de moment magnétique connu, que l'on fait tourner de $180^{\circ}$.

Dans les premières expériences, le champ a été mesuré en faisant tourner de $180^{\circ}$ les bobines induites dont l'aire avait été mesurée, le galvanomètre étant étalonné au préalable au moyen d'un solénoïde entouré de quelques tours du circuit induit. Plus tard, je me suis servi de la balance magnétique absolue de M. A. Cotton ('). Toutes les fois que le champ est suffisamment uniforme dans une étendue de 2 centimètres, cet appareil est d'une commodité et d'une exactitude remarquables.

Les sphères de pyrrhotine avaient $y$ millimètres de diamètre, les disques 9 millimètres de diamètre et des épaisseurs variant un peu d'un disque à l'autre, un peu inférieures à 1 millimètre.

\section{B. - Mesure du couple exercé par le champ sur le cristal.}

Après avoir fait par la méthode d'induction de nombreuses observations, je l'ai remplacée presque complètement par une méthode fondée sur la mesure du couple qui tend à faire tourner la substance autour d'un axe vertical quand un champ d'intensité déterminée occupe successivement tous les azimuts dans le plan horizontal. Ce couple est égal au champ multiplié par la composante de l'aimantation perpendiculaire au champ et horizontale. Le champ étant connu, cette composante l'est aussi par la mesure du couple.

Dans un certain nombre de questions, cette composante intervient seule, et la méthode se suffit à elle-même. Je montrerai dans le chapitre consacré au plan magnétique dans quelle mesure elle peut être adaptée à la mesure de la composante de l'aimantation parallèle au champ.

La $f g$. כ̊ représentel'un des appareils que j'ai employés. Un électroaimant $\mathrm{E}$, dont les bobines portent 2000 tours de fil, donne, entre les noyaux écartés de $12^{\mathrm{mm}}$,, , pour un courant de 1 ŏ ampères, un champ très uniforme de 11000 unités. Il est porté par l'intermédiaire d'une

(1) A. Cotron, J. de Phys., 3érie, IX, p. 383 ; 1900. 
PROPRIÉTÉS MAGNÉTIQUES DE LA PYRRHOTINE 479

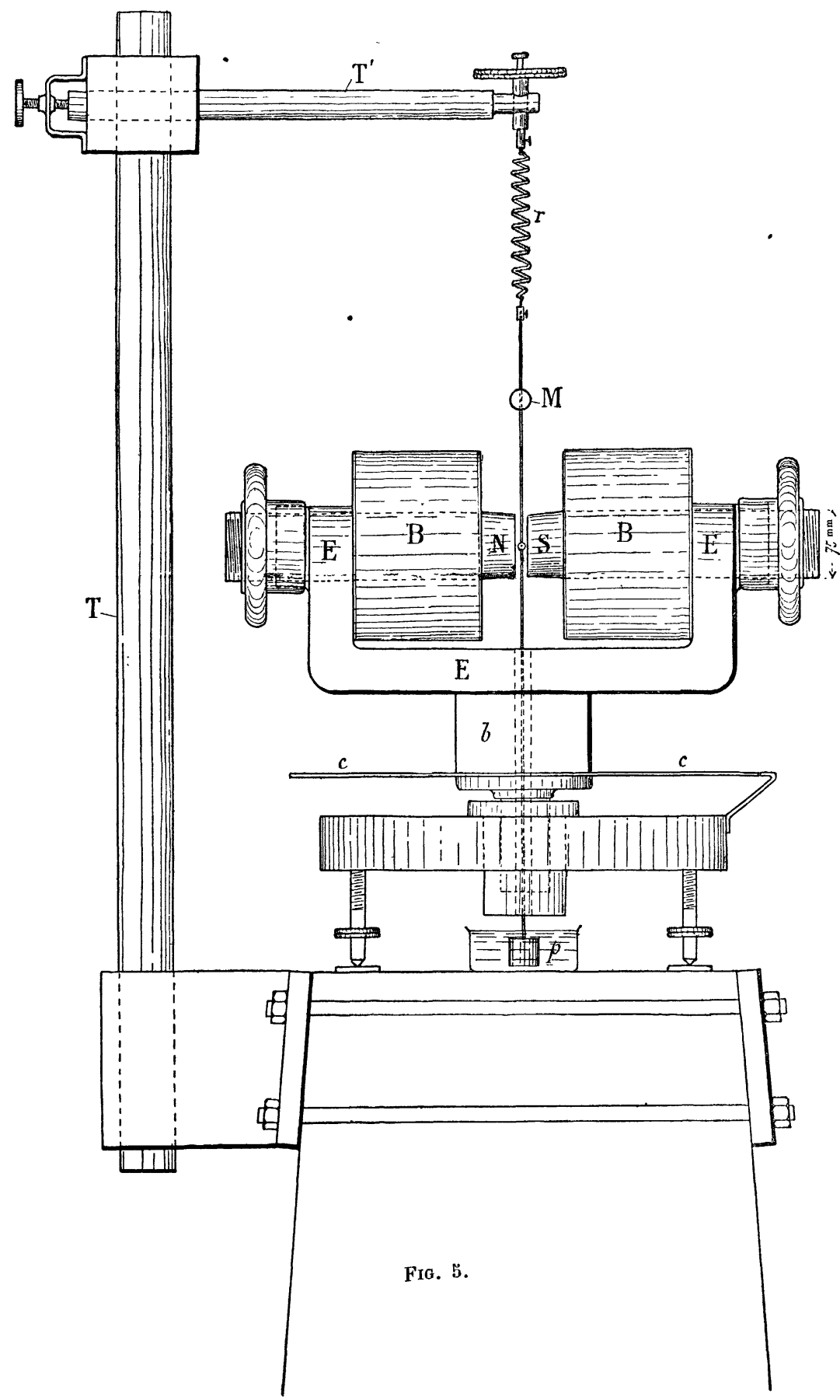


pièce de bois $b$, destinée à ménager la place nécessaire à la balance magnétique, par un pivot de bronze percé dans l'axe, et entraîne le cercle divisé $c$.

La substance placée entre les pôles est fixée sur une longue tige de cuivre suspendue au ressort de torsion $r$. Cette tige porte le miroir M, pour la lecture des déviations de l'équipage, et le poids de laiton $p$, plongé dans de la glycérine qui donne de la stabilité et sert d'amortisseur. Le bâti, formé de forts tubes de laiton $\mathrm{T}, \mathrm{T}^{\prime}$, est pourvu des mouvements de réglage nécessaires, dont une partie seule est représentée.

Cette méthode est beaucoup plus sensible que celle du galvanomètre balistique et permet d'examiner des échantillons beaucoup plus petits. Quand on se sert d'échantillons de même grandeur, toutes les perturbations dues aux courants d'air, au magnétisme du support sont négligeables; elle devient extrêmement commode et sûre.

La petite correction des azimuls du champ par rapport à la substance lus sur le cercle, provenant de ce que la substance tourne par la torsion du ressort, a été faite.

\section{CHAPITRE III. \\ ASSOCIATION DE TROIS CRISTAUX ÉlÉmentaines DANS LE PLAN MAgNÉtiQUe.}

J'ai montré antérieurement $\left({ }^{1}\right)$ que les cristaux de pyrrhotine ne s'aimantent que parallèlement au plan de base du prisme hexagonal que j'ai appelé plan magnétique ; je consacrerai un chapitre du présent travail à cette question. Mais auparavant, pour déblayer le terrain, il est nécessaire de parler des propriétés magnétiques dans ce plan. Elles ont eu d'abord une allure très énigmatique. Très variables d'un échantillon à l'autre, elles possédaient tout au plus la symétrie clinorhombique à la place de la symétrie hexagonale attendue. Cette confusion s'est dissipée tout à coup à l'examen des courbes ( $f g .6$ ), qui représentent un résultat immédiat d'expérience. Dans cette figure, les abscisses sont les azimuts d'un champ magnétique constant, et les crdonnées de la courbe supé-

(1) C. R.. t. CXXVI, p. $1099 ; 1899 ;-J$. de Phys., 3 série, t. VIII, p. 342; 1899. 
PROPRIÉTÉS MAGNÉTIQUES DE LA PYRRHOTINE 481

rieure les composantes de l'aimantation parallèle au champ, celles de la courbe inférieure les composantes perpendiculaires au champ. Les phénomènes se reproduisent à $180^{\circ}$ de distance.

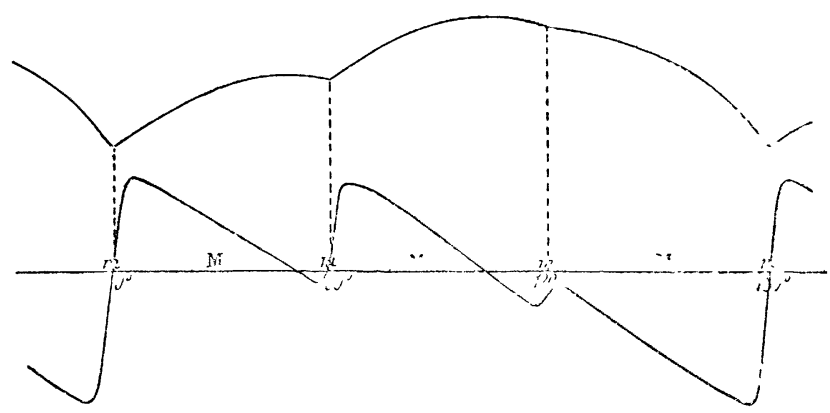

FIG. 6.

Pour interpréter cette courbe, j'ai recours à une image. Supposons que nous fassions tourner le champ magnétique dans le plan d'une plaque elliptique de fer doux. Aux axes, l'aimantation coïncidera avec le champ. Le grand axe sera un maximum, le petit axe un minimum d'aimantation. Pour toute autre direction du champ, l'aimantation sera plus voisine du grand axe que le champ. Dans la rotation continue du champ, l'aimantation tournera donc plus len-

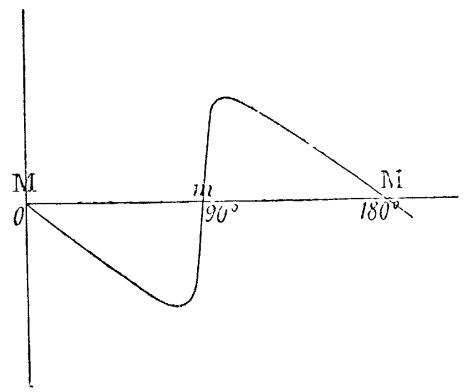

Fig. 7.

tement que celui-ci dans le voisinage du grand axe et plus vite dans le voisinage du petit axe. Si l'ellipse est très allongée, dans le voisinage du minimum, la composante de l'aimantation perpendiculaire au champ passera presque instantanément d'une très grande 
valeur négative à une très grande valeur positive, comme le représente la $f g .7$. La courbe inférieure de la $f g$. 6 résulte visiblement de l'addition des ordonnées de trois courbes analogues à celles de la fig. 7 , construites à des échelles différentes et déplacées les unes par rapport aux autres de $60^{\circ}$ et de $120^{\circ}$.

Nous supposerons donc que l'édifice complexe du cristal résulte. de la juxtaposition de cristaux élémentaires dont les plans magnétiques sont parallèles et qui possèdent chacun un maximum et un minimum d'aimantation rectangulaire. Ces cristaux sont associés dans le plan magnétique sous des angles de $60^{\circ}$, ou, ce qui revient au même, de $120^{\circ}$.

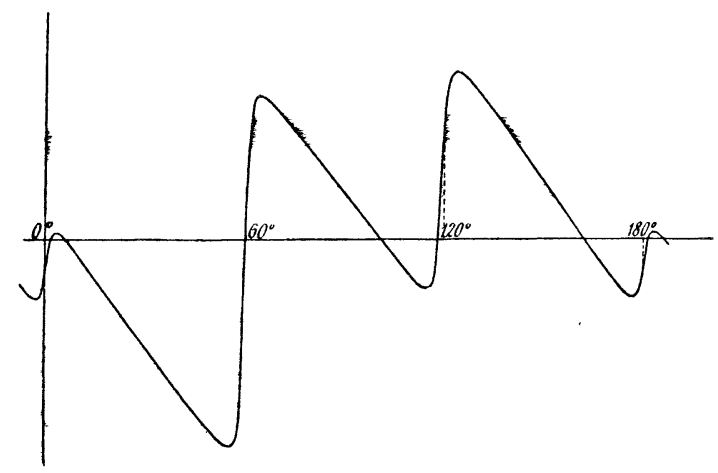

FIG. 8.

L'amplitude des variations brusques de la composante perpendiculaire au champ donne l'importance relative des trois composantes. Cette analyse du groupement cristallin se fait avec une netteté inégale suivant la grandeur du champ employé. La fig. 8, représentant l'aimantation perpendiculaire au champ pour une substance de Morro Velho dans un champ de 4000 gauss, donne un exemple de phénomène particulièrement brusque. La répartition de la matière entre les trois composantes est donnée dans cet exemple par :

$$
100: 66,1: 16,9 \text {. }
$$

Les angles rentrants de la courbe supérieure de la fig. 6 correspondent aux minima très accentués de l'aimantation parallèle au ohamp de chacune des composantes.

Cherchant à isoler la pyrrhotine élémentaire, j'ai déterminé la 
PROPRIÉTÉS MAGNÉTIQUES DE LA PYRRHOTINE 483 composition de plusieurs centaines d'échantillons. Pour certains d'entre eux, les trois composantes étaient sensiblement égales ; pour d'autres, deux d'entre elles prédominaient ; pour d'autres encore, une seule était prépondérante. Mais, même en divisant la matière en tout petits fragments, je n'ai pu rencontrer un échantillon absolument simple. La subdivision parallèle ou perpendiculaire au plan magnétique semblait indifféremment peu avantageuse dans cetle recherche. On peut en conclure que l'enchevêtrement est très intime et que la composition, peu variable, a été déterminée, sans doute, par les conditions à peu près uniformes dans toute son étendue dans lesquelles le cristal s'est formé. Avec de la patience, on trouve des fragments relativement simples. Ainsi j'ai mesuré sur un petit clivage de New-Jersey, dont j'ai fait une étude magnétique complète:

Substance 1 (New-Jersey), les rapports

$$
100: 4,75: 3,1 \text {. }
$$

Sur un autre échantillon, de $0^{\text {mgr }}, 4$, trop petit pour des mesures précises, j'ai évalué :

$$
100: 3: 0 \text {. }
$$

L'existence de la matière simple est donc certaine.

J'indiquerai encore ici la composition de deux échantillons sur lesquels j'ai fait de nombreuses mesures:

Substance 2 (New-Jersey) :

$100: 11: 4,7$.

Une deuxième détermination, fondée sur des expériences nombreuses faites avec des appareils différents plus précis, a donné :

$$
100: 10,85: 4,95 .
$$

Substance 3 (Morro Velho), premières expériences imparfaites, méthode balistique:

$$
100: 17,5: 5,5 .
$$

Même substance, huit ans plus tard, méthode de mesure des couples, expériences précises :

$$
100: 19,6: 5,4 .
$$


C'est cette dernière substance (disque de 9 millimètres de dia-

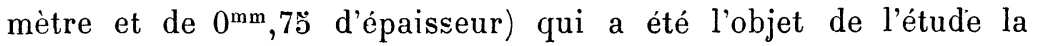
plus complète.

On voit par ces exemples que la détermination de la composition d'un cristal de pyrrhotine se fait avec une certaine sûreté, que j'évalue, dans les meilleurs cas, à $0,20 / 0$.

Ne pouvant isoler l'élément simple, je me suis résigné à déduire ses propriétés de celles des cristaux complexes. J'ai déterminé à cet effet par tâtonnements les deux coefficients par lesquels il faut multiplier les résultats d'observation pour les retrancher ensuite, avec une avance de $60^{\circ}$ ou de $120^{\circ}$, afin que les ordonnées restantes représentent les propriétés d'un cristal simple. La fig. 9, qui se rap-

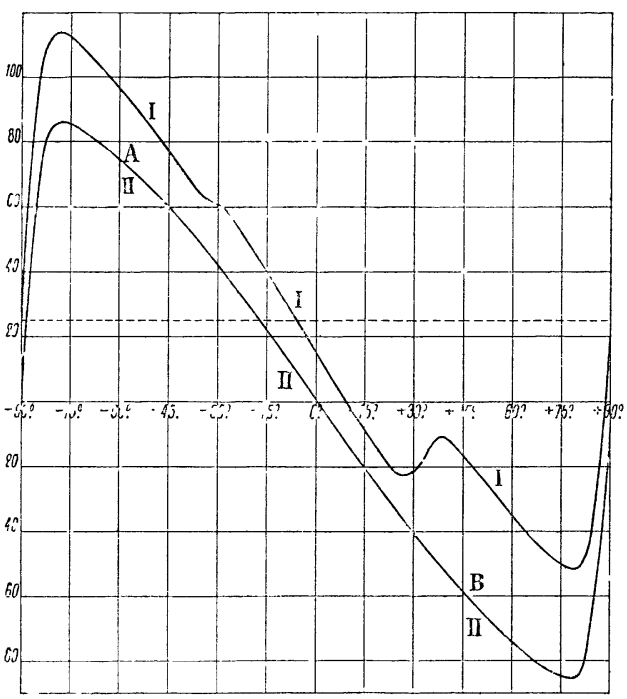

FIG. 9.

porte à la substance 3 , dans un champ de 1992 gauss, donne un exemple de cette épuration graphique. La courbe I est expérimentale, la courbe Il est corrigée des deux substances parasites. Le critérium de la réussite de l'opération est la régularité de la courbe entre $\mathrm{A}$ et B, qui s'estime très sûrement, grâce à sa forme presque rectiligne. La composante de l'aimantation parallèle au champ est traitée de la même manière que la composante de l'aimantation perpendicu- 
PROPRIÉtÉs MAgNétiques DE LA PYRRHOTINE 485

laire au champ, et ces opérations sont répétées pour plusieurs valeurs du champ. Le résultat de ces opérations sera décrit au chapitre suivant; il est compatible avec la symétrie orthorhombique.

Les propriétés ferromagnétiques, dont la connaissance est ici le but du travail, peuvent donc, dans certains cas, être un moyen d'investigation des groupements cristallins. La portée de ce procédé cst limitée par le petit nombre des substances ferromagnétiques. Mais, comme elles sont opaques, il peut rendre des services précisément là où la méthode optique est impuissante.

\section{CHAPITRE IV.}

PROPRIÉTÉS DU CRISTAL ÉLÉMENTAIRE.

Soient, dans un cristal élémentaire de pyrrhotine, $\mathrm{H}_{x}, \mathrm{H}_{y}$, les deux composantes du champ; $\mathrm{I}_{x}, \mathrm{I}_{y}$, celles de l'intensité d'aimantation dans le plan magnétique, l'axe $\mathrm{O} x$ (fig. 10) étant la direction de facile aimantation, laxe $\mathrm{O} y$ celle de difficile aimantation. Lorsque l'extrémité du vecteur $\mathrm{H}$ décrit le plan, l'expérience montre que l'extrémité du vecteur I reste à l'intérieur d'une circonférence E, que j’appellerai le cercle de saturation, dont le rayon $I_{M}$ est l'intensité de l'aimantation à saturation.

Si nous faisons abstraction des phénomènes d'hystérèse, il est indifférent que $\mathrm{H}$ arrive en un point par un chemin ou par un autre. On obtiendra donc tous les renseignements désirables en opérant avec des champs constants, de différentes grandeurs, tournant dans le plan magnétique.

La fig. 10 montre la physionomie des courbes A, B, C, D que décrit le vecteur I quand le champ a les valeurs de 1992, 4000, 7310, 11140 gauss. Pour tous ces champs, l'aimantation atteint la valeur $\mathrm{I}_{\mathrm{M}}$ dans la direction $\mathrm{O} x$, et, quand le champ s'écarte de $\mathrm{O} x$, suit d'abord le cercle de saturation, sur un arc d'autant plus étendu que le champ est plus intense. Puis elle quitte assez brusquement ce cercle, pour décrire une courbe assez voisine d'une corde parallèle à $\mathrm{O} x$.

La rotation de l'aimantation est, dans un angle assez étendu à partir de $\mathrm{O} x$, proportionnelle à la rotation du champ, mais d'autant 
plus ralentie par rapport à celle-ci que le champ est plus faible. Dans le voisinage de $\mathrm{O} y$, la rotation de l'aimantation est, au contraire, beaucoup plus rapide que celle du champ, et le rapport des tangentes des angles de l'aimantation et du champ avec $\mathrm{O} y$ est sensiblement constant.

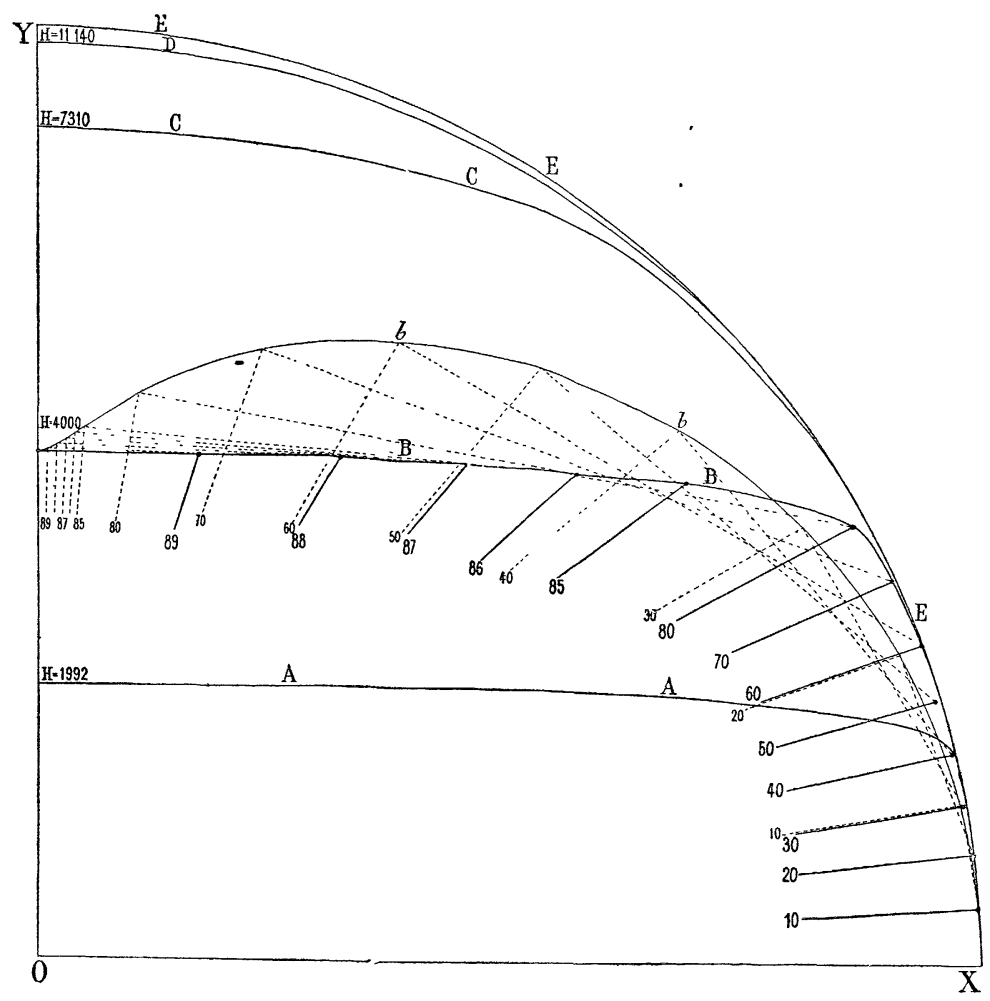

FIG. 10.

Ces caractères ont été rendus sensibles dans la courbe B, correspondant à $\mathrm{H}=4000$ gauss, dans laquelle les rayons en traits pleins représentent les directions de l'aimantation, les rayons pointillés les directions du champ, les nombres indiquant, pour les uns et les autres, les angles correspondants du champ avec $\mathrm{O} x$.

Pour construire cette courbe, j'ai porté d'abord dans la direction des champs, pour tous les angles de $10^{\circ}$ en $10^{\circ}$ dans le voisinage du maximum, de degré en degré dans le voisinage du minimum, 
PROPRIÉTÉS MAGNÉTIQUES DE LA PYRRHOTINE 487 la composante de l'aimantation parallèle au champ. J'ai obtenu ainsi la courbe $b$ de la composante de l'aimantation parallèle au champ; j'en ai ensuite déduit la courbe $B$ en portant l'aimantation perpendiculaire au champ perpendiculairement sur les rayons vecteurs de la courbe $b$. Les données d'expérience relatives à cette construction, pour cinq valeurs différentes du champ, sont contenues dans les fig. 11 et 12.

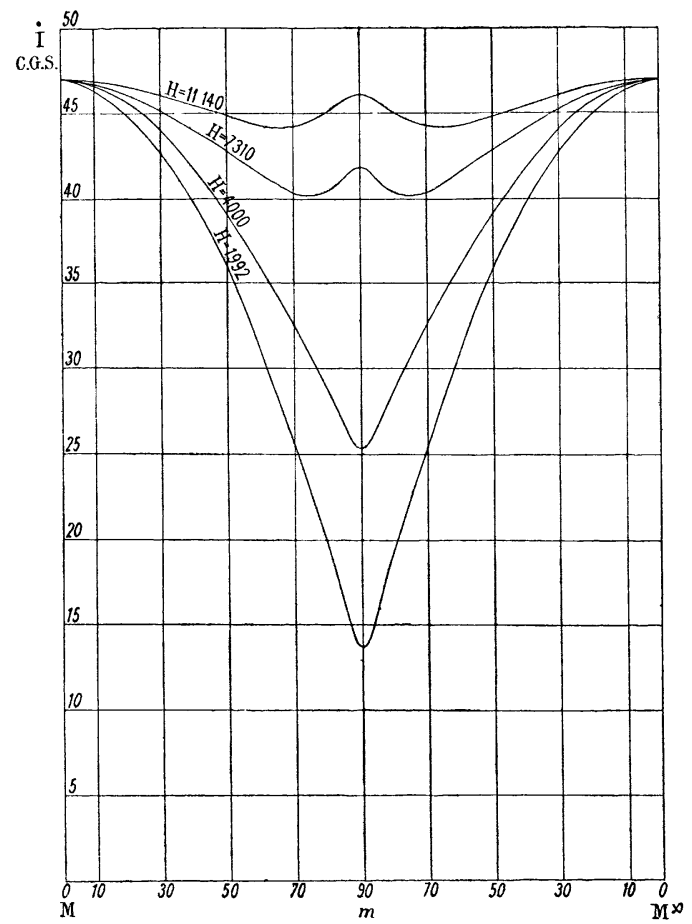

Fig. 11. - Composante de l'aimantation parallèle au champ.

Cette physionomie générale appartient à toutes les pyrrhotines, que nous distinguerons plus tard en normales et anormales, et à toutes les valeurs du champ, depuis les plus faibles jusqu'à celles qui suffisent pour faire décrire à I le cercle de saturation tout entier. Ce qui suit, par contre, est particulier aux pyrrhotines normales de Morro-Velho en cristaux compacts.

Soient $\alpha$ et $p$ les angles de $\mathrm{H}$ et de I avec $\mathrm{O} x(f g .13)$; décomposons $\mathrm{H}$ en deux composantes $\mathrm{H}_{\mathrm{D}}$ dirigée suivant $\mathrm{O} y$ et $\mathrm{H}_{\mathrm{I}}$ parallèle à l'inten- 


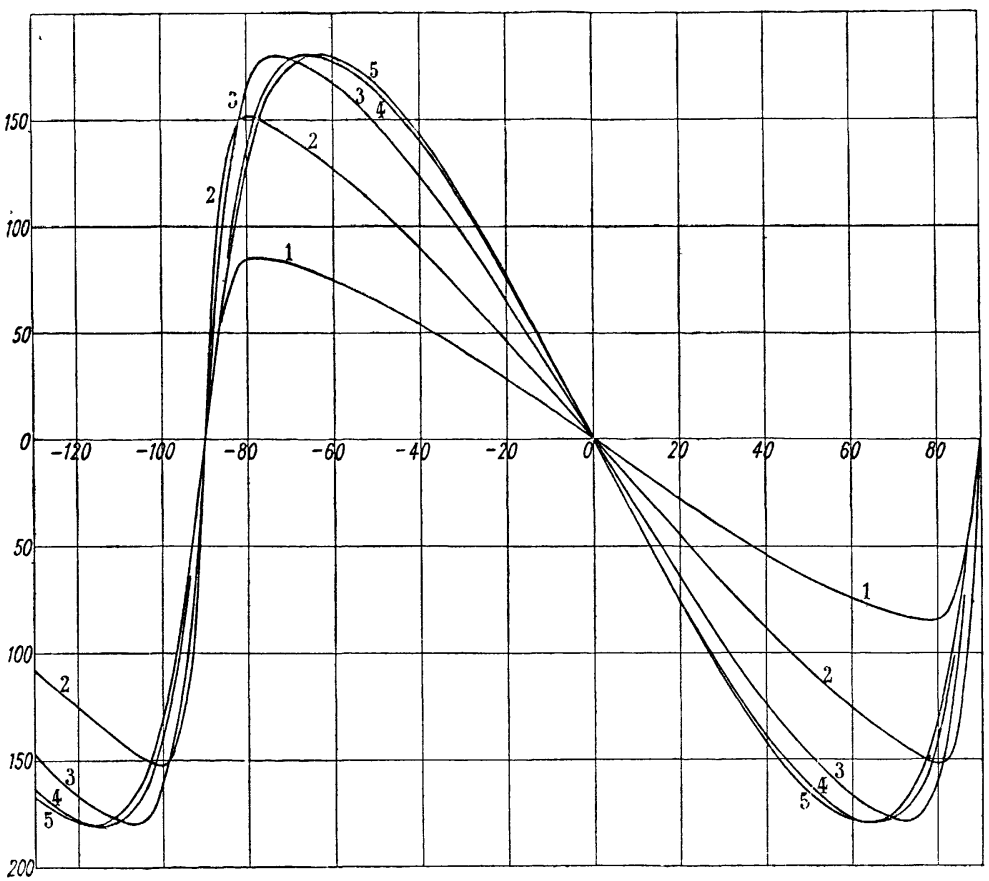

FIG. 12.

Courbe $1 \ldots \ldots \quad \mathrm{H}=1992$ gauss

- 2.... $\mathrm{H}=4000$

$-3 \ldots \ldots \quad \mathrm{H}=7310$

$-4 \ldots \ldots \quad \mathrm{I}=10275$

- 5.... $\mathrm{H}=11140$

Abscisses: Angles d'orientation du champ par rapport à la substance, mesurés dans le plan magnétique.

Ordonnées : Couples en millimètres de l'échelle divisée. Pour obtenir ces couples en ergs par centimètre cube de la substance, multiplier par 950.

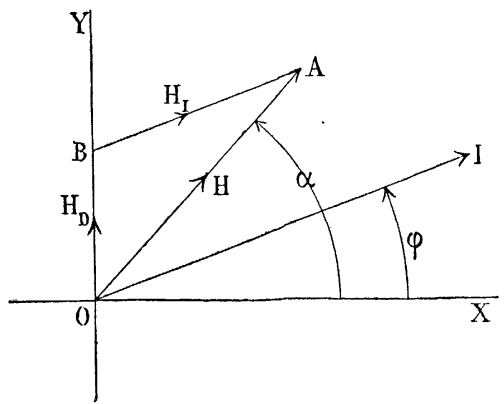

FIG. 13. 
PROPRIÉTÉS MAGNÉTIQUES DE LA PYRRHOTINE 489 sité d'aimantation. Il y a un rapport constant

$$
\frac{\mathrm{H}_{\mathrm{D}}}{\mathrm{I} \sin \varphi}=\mathrm{N}
$$

entre la composante $H_{D}$ du champ et la composante de l'aimantation suivant $\mathrm{O} y$, quels que soient $\mathrm{H}$ et $\alpha$.

Cette proposition est établie par la fig. 14, dans laquelle j'ai porté en abscisses les champs $H_{D}$ résultant de cette décomposition et en ordonnées les composantes I sin §, pour les cinq séries d'expériences faites avec les champs de 1992, 4000, 7310, 1027\%, 11140 gauss, en déplaçant pour chaque série l'origine sur l'axe des abscisses d'une quantité égale à õ00 gauss, pour éviter la superposition des courbes. Les nombres portés sur les courbes sont les valeurs de $\alpha$ en degrés. Les droites pointillées correspondent à

$$
\frac{\mathrm{H}_{\mathrm{D}}}{\mathrm{l} \sin \varphi}=\frac{7300}{47}
$$

elles coïncident au degré de précision des expériences avec les courbes. Tout se passe donc comme si la substance annulait, par un phénamène démagnétisant dî̀ $\dot{a}$ sa structure, une composante du champ proportionnelle à la composante de l'aimantation dans la direction de difficile aimantation, et qu'ensuite la composante restante était parallèle à l'aimantation.

Dans l'échantillon examiné, $\mathrm{I}_{M}$ est égal à 47 unités. Le champ démagnétisant maximum est de 7300 gauss. $I_{M}$ est assez variable d'un échantillon à l'autre. Le champ démagnétisant maximum n'a été mesuré avec précision que sur un seul échantillon; je ne saurais donc dire s'il est une constante caractéristique de la pyrrhotine; mais il semble, d'après les propriétés de quelques autres échantillons examinés plus sommairement, qu'il ne doive pas varier beaucoup.

Pour déduire de ce résultat expérimental les conséquences qu'il comporte, il est commode de faire intervenir la notion de travail d'aimantation. Ce travail est donné par la différentielle exacle :

$$
d \mathrm{~T}=\mathrm{H} \cos (\alpha-\varphi) d \mathrm{I}+\mathrm{HI} \sin (\alpha-\varphi) d \varphi,
$$

où $\mathrm{H}$ et $\alpha$ sont considérés comme fonctions des variables indépendantes $\mathrm{I}$ et $\stackrel{\text {. Il }}{\mathrm{I}}$ en résulte :

$$
\frac{\partial H \cos (\alpha-\varphi)}{\partial \varphi}=\frac{\partial H I \sin (\alpha-\varphi)}{\partial I} .
$$




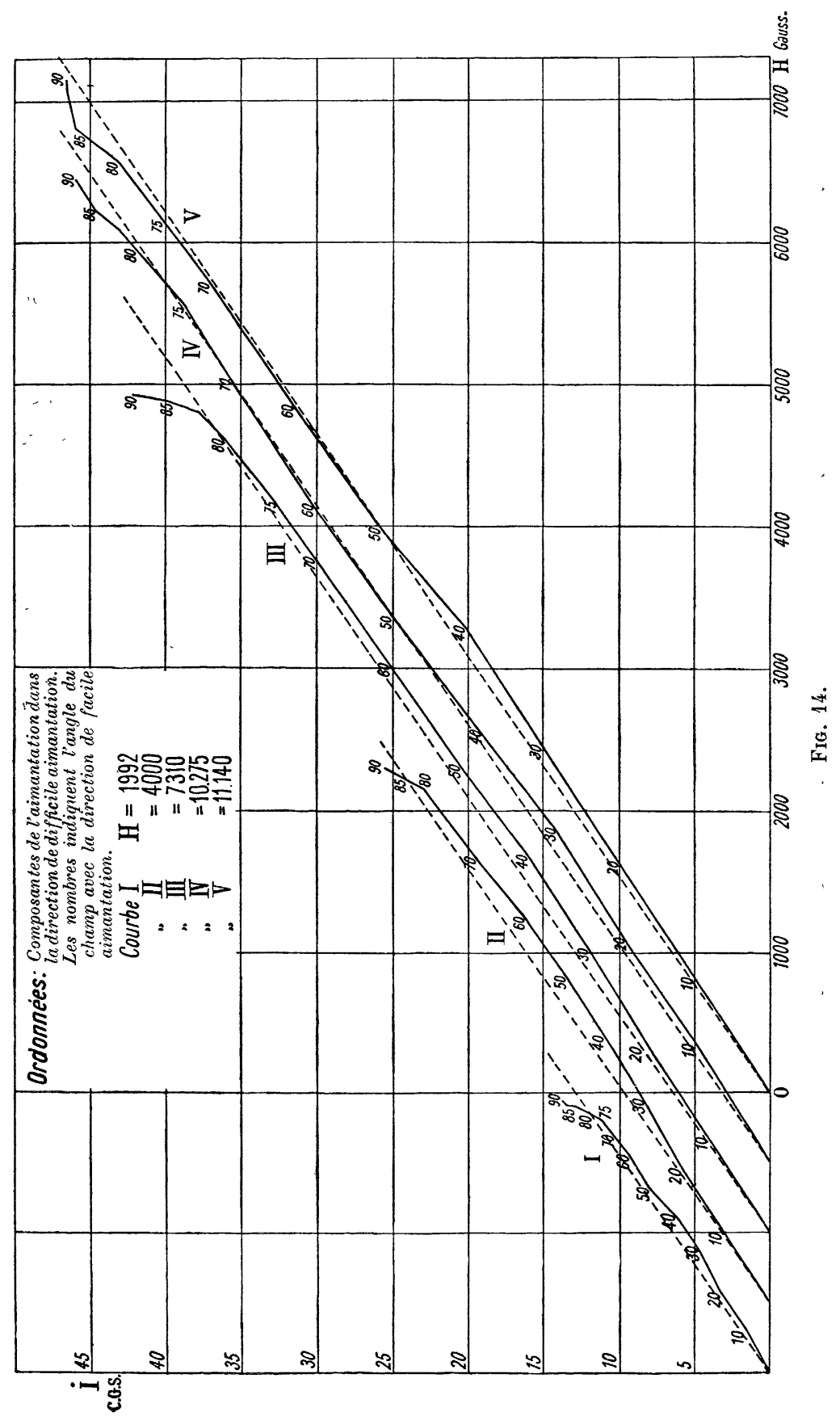




\section{PROPRIÉTÉS MAGNÉTIQUES DE LA PYRRHOTINE 491}

$\mathrm{Si}$, pour une substance, laimantation a constamment la direction du champ, $\alpha-\vartheta=o$, et par suite $\frac{\partial H}{\partial \varphi}=0$. La relation entre le champ et l'intensité d'aimantation est donc la même dans tous les azimuts.

Cela posé, remarquons que le travail de la composante du champ $\mathrm{H}_{\mathrm{D}}=\mathrm{NI} \sin \varphi$, considérée seule, est NI $\sin \varphi d \mathrm{I} \sin \varphi$, c'est-à-dire différentielle exacte. Il s'ensuit que le travail de l'autre composante H l'est aussi. Donc la proposition que nous venons d'établir s'applique à cette composante. Nous en concluons que la loi de l'aimantation, déduction faite du champ démagnétisant $\mathrm{H}_{\mathrm{D}}$, est la même dans tous les azimuts.

Reste à déterminer cette loi pour un azimut particulier. Je choisis à cet effet la direction de facile aimantation. La courbe $\mathrm{OAC}$ de la fig. 15, page 493, représente l'intensité d'aimantation en fonction du champ pour cette direction.

Elle part de l'origine avec une inclinaison constante, correspondant à une susceptibilité initiale $k_{0}=\frac{47}{110}$, qui la conduirait, si elle se continuait, à la saturation pour un champ de 110 gauss. Au lieu de cela, la courbe devient concave du côté de l'axe des abscisses, à partir d'une intensité égale à $\frac{I_{M}}{2}$ environ, et s'arrondit vers la ligne de saturation $\mathrm{I}_{\mathrm{M}}=$ constante, à laquelle elle est tangente à l'abscisse de 700 gauss. A partir de ce moment, elle se confond exactement avec elle jusqu'aux champs les plus élevés dans tout l'intervalle accessible, jusqu'à 11140 gauss. Un exemple aussi caractéristique de saturation constante ne se rencontre pas, à ma connaissance, parmitoutes les données expérimentales que l'on possède sur les corps ferromagnétiques.

Pour l'interprétation des résultats expérimentaux dans les champs faibles, il convient de tenir compte des phénomènes démagnétisants dont l'objet en expérience est le siège, par suite de ses dimensions finies. Celui-ci, un disque de 9 millimètres de diamètre et de $0^{\mathrm{mm}}, 75$ d'épaisseur, donnerait, assimilé à l'ellipsoïde aplati inscrit, par suite du phénomène démagnétisant, une susceptibilité initiale apparente égale à 1,5 seulement, alors même que la substance dont il serait fait aurait une susceptibilité infinie. Elle est un peu plus forte que celle dudisque de pyrrhotine. Mais l'écart entre les deux se réduit singulièrement si l'on considère que les cristaux ne sont jamais tout à fait 
exempts d'impuretés, que leur matière n'est souvent pas tout à fait continue, comme en témoignent les variations de la densité et de petites cavités limitées souvent par les faces naturelles du cristal, et que l'échantillon actuel en particulier était sillonné de petites cassures assez nombreuses. Remarquons en outre que, si la substance avait la forme d'un ellipsoïde et qu'elle élait aimantée dans la direction de l'un de ses axes, la loi démagnétisante serait rectiligne jusqu'à la saturation. Par contre, si, pour l'ellipsoïde, la direction du champ est quelconque et si l'on considère la composante de l'aimantation dans la direction du champ, la loi d'aimantation apparente d'une substance de susceptibilité infinie n'est pas linéaire; elle tend asymptotiquement vers la saturation $\left({ }^{1}\right)$; sa susceptibilité apparente sera décroissante vers la saturation. Les défauts de la matière donnent lieu précisément à des champs démagnétisants obliques sur l'intensité d'aimantation.

Rien ne s'oppose donc à ce que l'on fasse l'hypothèse que, la substance étant supposée parfaitement continue et indéfinie, l'aimantation atteint, dans la direction de facile aimantation, la saturation dès les champs les plus faibles et la conserve absolument constante jusqu'aux champs les plus élevés.

Cette hypothèse est certainement la plus simple que l'on puisse imaginer; elle doit donc être adoptée jusqu'à ce qu'elle ait été mise en contradiction avec l'expérience. Elle peut sans doute être étendue dans certains cas aux métaux et expliquerait alors aisément, pour les fils de fer dont la structure fibreuse est certainement très continue dans le sens longitudinal, les perméabilités atteignant 60000 trouvées par Ewing, quand des trépidations suppriment les phénomènes d'hystérèse.

Cette hypothèse permet de donner une définition de la courbe d'aimantation réversible ou courbe d'équilibre. Ce serait la courbe d'aimantation d'une matière fictive, de même structure que la matière réelle, mais de susceptibilité infinie et dépourvue d'hystérèse. Elle fournit ainsi un point d'appui à la discussion de la question sur laquelle M. Ch. Maurain a fait récemment d'intéressantes expériences $\left({ }^{2}\right)$.

L'expérience donne autant de vérifications qu'il y a de directions : pour lesquelles la loi d'aimantation a été déterminée. Considérons

(1) Voir, dans le mème ordre d'idées, les considérations de la page 494.

(2) J. de Phys., $4^{\mathrm{c}}$ série, t. III ; p. 417. 
la courbe $\mathrm{OB}^{\prime} \mathrm{C}^{\prime}(f g, 1 \breve{3})$, correspondant à la direction de difficile aimantation. Si la matière était parfaitement continue et illimitée, elle devrait coïncider avec la ligne brisée $\mathrm{OBC}$, tracée en donnant au point $\mathrm{B}$ une abscisse égale au champ démagnétisant maximum. de 7300 gauss.

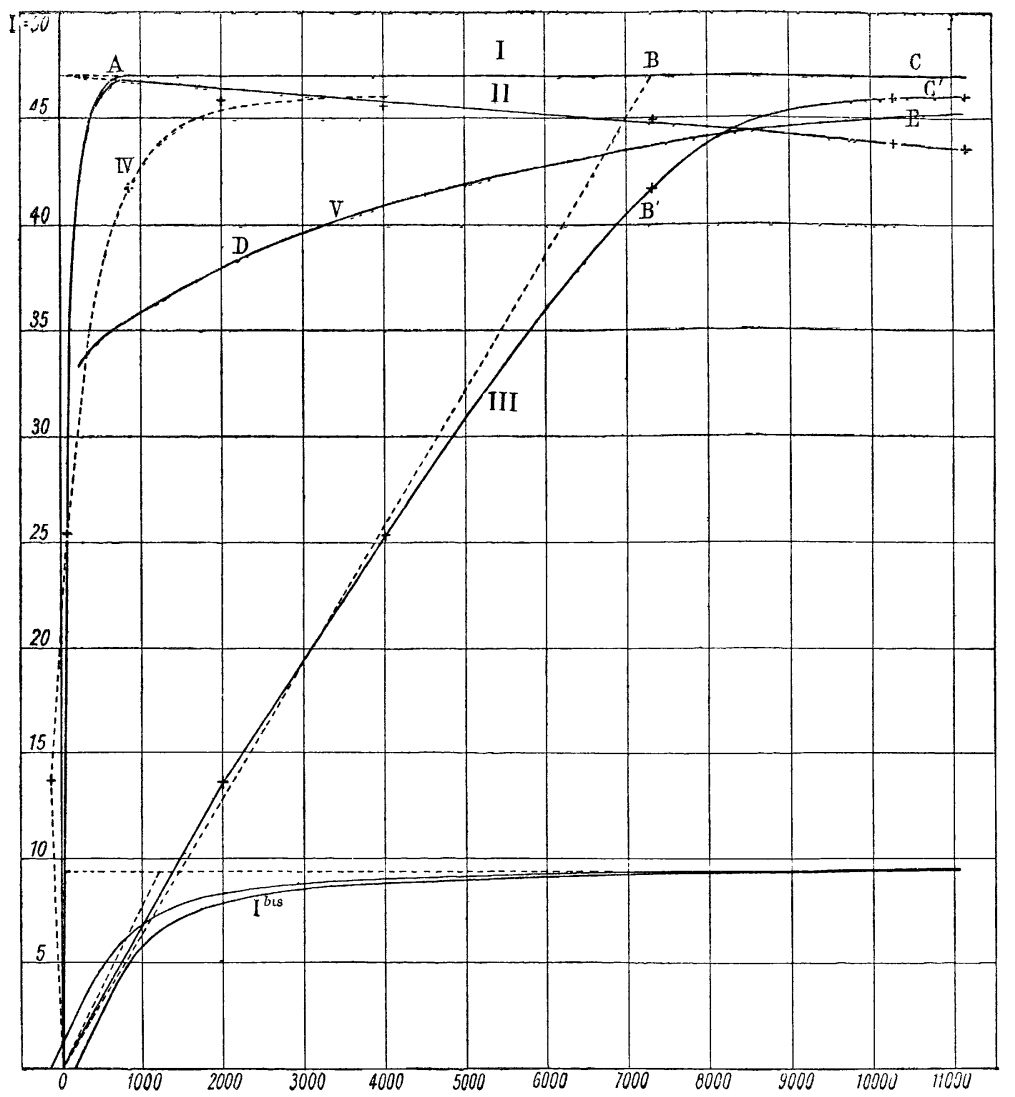

FIG. 15.

Courbes d'aimantation. - I, dans la direction de facile aimantation. - II, comme courbe I, mais sans la correction de susceptibilité normale. - III, dans la direction de difficile aimantation. - IV et V, voir le texte. - I bis, comme I, échelle du champ dix fois plus grande, de l'aimantation cinq fois plus petite.

Si la condition d'isotropie après déduction du champ démagnétisant était satisfaite, il devrait y avoir la même différence d'abscisses entre $O B^{\prime} \mathrm{C}^{\prime}$ et la ligne brisée $\mathrm{OBC}$ qu'entre $\mathrm{OA}$ et l'axe des ordon- 
nées. Ces premières différences sont un peu plus grandes en général, pas assez cependant pour altérer le caractère de la courbe $\left(0 \mathrm{~B}^{\prime} \mathrm{C}^{\prime}\right.$. La divergence ne dépasse pas l'incertitude expérimentale, qui est assez grande au minimum, à cause des variations rapides des deux composantes de l'aimantation en fonction de $\alpha$. Pour faciliter la comparaison, la courbe $\mathrm{OB}^{\prime} \mathrm{C}^{\prime}$, déduction faite des champs démagnétisants, a été tracée en IV.

La pyrrhotine nous fournit donc, au maximum et au minimum, deux types de "courbes d'aimantation" essentiellement différents, mais qui ont un caractère commun : la saturation est atteinte pour une valeur finie du champ. L'aimantation à saturation au minimum a été trouvée inférieure de $2 \mathrm{p}$. cent à cette quantité au maximum. Cette faible différence est due sans doute à des erreurs d'expériences. Je n'ai pas réussi à en préciser la nature, bien que cet écart soit supérieur à l'incertitude moyenne des mesures.

Pour une direction oblique sur les axes, plusieurs définitions de la courbe d'aimantation sont possibles ; je considérerai ici, pour un champ de direction constante, la composante de l'aimantation dans cette direction en fonction de l'intensité de ce champ. L'aimantation ne coïncidant en direction avec le champ que quand il est infini, cette courbe d'aimantation aura la droite de saturation comme asymptote.

Cherchons l'équation de cette courbe. On déduit du triangle OAB (fig. 13) comme expression de la loi démagnétisante :

$$
\mathrm{H} \sin (\alpha-\varphi)=\mathrm{NI} \sin \varphi \cos \varphi,
$$

qui, appliquée au cercle de saturation, devient :

$$
\mathrm{H} \sin (\alpha-\varphi)=\mathrm{NI}_{\mathrm{M}} \sin \varphi \cos \varphi \text {. }
$$

Et posons :

$$
\Im=\mathrm{I}_{\mathrm{M}} \cos (\alpha-\varphi),
$$

on obtient par élimination de $\varphi$ entre ces deux dernières équations :

$$
\mathrm{H}=\frac{\mathrm{N}}{2} \sin 2 \alpha \frac{\jmath^{2}}{\sqrt{\mathrm{I}^{2} \mathrm{M}-\jmath^{2}}}-\mathrm{N} \jmath \cos 2 \alpha-\frac{\mathrm{N}}{2} \sin 2 \alpha \sqrt{\mathrm{IM}^{2}-\jmath^{2}} .
$$

Le premier seul des trois termes du second membre devient infini quand $J$ tend vers $I_{M}$. Quand $\frac{1}{H}$ est infiniment petit du premier 
PROPRIÉTÉS MAGNÉTIQUES DE LA PYRRHOTINE 495 ordre, $I_{M}-ð$ est infiniment petit du second ordre. Cette propriété est accessible à l'expérience, mais d'une vérification délicate.

La courbe $\mathrm{V}$ de la figure 15 représente la courbe d'aimantation au sens ci-dessus indiqué, pour $\varphi=45^{\circ}$.

On conçoit aisément que toutes les courbes d'aimantation usuelles puissent s'expliquer par la superposition de propriétés analogues à celles de la pyrrhotine dans la direction du maximum, du minimum et dans les directions obliques.

La nécessité de multiplier les contrôles conduit à envisager les hypothèses démagnétisantes que nous avons faites sous d'autres aspects.

a) Définissons, par analogie avec $\mathrm{N}=\frac{\mathrm{H}_{\mathrm{D}}}{\mathrm{I} \sin \varphi}$, un coefficient :

$$
n=\frac{\mathrm{H}_{\mathrm{I}}}{\mathrm{I}}
$$

$n$ est l'inverse de la susceptibilité relative à l'aimantation rendue isotrope par la défalcation du champ $\mathrm{H}_{\mathrm{D}}$. Tant que $\mathrm{I}$ est petit, la susceptibilité initiale $\frac{1}{n}$ est constante; $n$ est déjà connu par la courbe d'aimantation relevée dans la direction de facile aimantation et est égal à :

$$
\frac{110}{47}=2,34 \text {. }
$$

En déduisant $n$ de données provenant du voisinage du minimum d'aimantation, on vérifie l'isotropie de la substance après déduction du champ démagnétisant NI.

Or on démontre facilement qu'au minimum, tant que la saturation n'est pas atteinte, le rapport de la vitesse de rotation de l'aimantation à celle du champ est :

$$
\frac{\Delta \varphi}{\Delta \alpha}=\frac{N}{n}+1
$$

Cette quantité est assez facile à relever aver, une certaine exactitude sur les courbes telles que celles de la fig. 10; on trouve :

$\begin{array}{ccc}\mathrm{H} & \frac{\Delta \varphi}{\Delta \alpha} & n \\ 97,2 & 82 & 1,92 \\ 194 & 77 & 2,05 \\ 564 & 73 & 2,13 \\ 2250 & 33 & 4,72 \\ 3830 & 17 & 9,15\end{array}$


On voit qu'en effet les valeurs de $n$ déduites des observations au minimum sont assez constantes dans les champs faibles et voisines de la valeur trouvée au maximum. Dans les champs plus forts, $n$ augmente comme cela est prévu.

b) Comme nous l'avons déjà indiqué, la relation

$$
\mathrm{H} \sin (\alpha-\varphi)=\mathrm{NI} \sin \varphi \cos \varphi,
$$

déduite du triangle $\mathrm{OAB}(f y .13)$, est l'expression analytique de la loi démagnétisante. Cette expression, multipliée par I, donne le couple exercé par le champ sur la substance :

$$
\mathrm{C}=\mathrm{HI} \sin (\alpha-\varphi)=\mathrm{NI}^{2} \sin \varphi \cos \varphi ;
$$

ce couple est maximum quand $I=I_{M}$ et $\varphi=45^{\circ}$. On doit donc, dans la mesure de $\mathrm{C}$ en fonction de $\alpha$, trouver un couple maximum invariable dès que le champ est assez fort pour faire décrire à l'aimantation, à partir de la direction $\mathrm{O} x$, un arc égal ou supérieur à $4 \mathrm{4}^{\circ}$ sur le cercle de saturation. C'est effectivement ce qui frappe à première vue dans la fig.12, dans laquelle le maximum des trois courbes correspondant aux champs les plus intenses est le même. Ces couples sont, exprimés en millimètres de l'échelle des déviations :

$$
\begin{aligned}
\text { Pour } \mathrm{H} & =4000 & & 149 \text { millimètres } \\
& =7310 & & 179,4 \\
& =10250 & & 181,0 \\
& =11140 & & 180,8
\end{aligned}
$$

Pour ramener ces couples à leurs valeurs absolues pour l'unité de volume, il faut les multiplier par $\frac{4 \bar{i}}{0,0494}$.

La valeur du couple maximum étant $\frac{\mathrm{NI}_{\mathrm{M}}{ }^{2}}{2}$ d'après $(3)$, on déduit de la moyenne des trois déterminations le champ démagnétisant maximum :

$$
\mathrm{NI}_{\mathrm{M}}=7300 .
$$

C'est cette valeur que jai admise comme étant la plus sûre, elle concorde bien avec l'ensemble des déterminations de la fig. 14, qui aurait permis d'hésiter entre 7200 et 7000 .

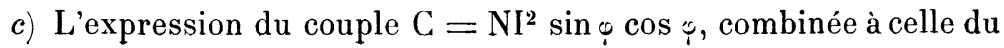


PROPRIÉtés MAgNétiQUES DE LA PYRRHOTINE 49 i rapport des vitesses de rotation de l'aimantation et du champ au minimum :

$$
\frac{\Delta \varphi}{\Delta \alpha}=\frac{N+n}{n}
$$

donne, pour le minimum,

$$
\frac{\Delta \mathrm{C}}{\Delta \alpha}=\mathrm{NI}^{2} \frac{\mathrm{N}+n}{n},
$$

et comme au minimum $(\mathrm{N}+n) \mathrm{I}=\mathrm{I}$,

$$
\frac{\Delta \mathrm{C}}{\Delta \alpha}=\mathrm{H}^{2} \frac{1}{n} \cdot \frac{1}{1+\frac{n}{\mathrm{~N}}}
$$

formule qui n'est plus valable quand la saturation est alteinte, parce qu'alors $\mathrm{N}$ et $n$ sont indéterminés, où :

$$
\frac{\Delta \mathrm{C}}{\Delta x}=\frac{\mathrm{H}^{2}}{n}
$$

avec une grande approximation.

$\frac{\Delta \mathrm{C}}{\Delta \alpha}$ est le coefficient angulaire au minimum de la courbe représentant les couples en fonction des azimuts, c'est-à-dire une donnée expérimentale immédiate. Si $n$ était constant, ce coefficient angulaire croîtrait proportionnellement au carré du champ. C'est là l'origine de la variation brusque qui a servi à analyser le groupement cristallin dans le plan magnétique. On pourra donc constater à l'aide de ce coefficient angulaire la constance de $n$.

Les expériences au maximum montrent qu'après une valeur initiale constante $n$ doit croître dans les champs plus élevés. On ne doit donc s'attendre à vérifier la constance de $\frac{1}{\mathrm{H}^{2}} \frac{\Delta \mathrm{C}}{\Delta \alpha}$ que pour les champs faibles. En outre, c'est le propre des erreurs expérimentales d'atténuer la brusquerie des phénomènes. Tous les petits accidents, tels que les déplacements angulaires provenant des petites cassures de la matière, la non-uniformité des champs démagnétisants, en retardant le phénomène pour certaines parties de la substance par rapport à d'autres, diminuent le maximum de $\frac{\Delta \mathrm{C}}{\Delta \alpha}$, et pour la courbe 
de l'aimantation parallèle au champ atténuent les angles rentrants. Aussi ai-je choisi parmi les échantillons dont je disposais, malgré sa moindre simplicité cristallographique, un disque donnant des phénomènes particulièrement brusques. J'ai trouvé au minimum de sa composante la plus importante

\begin{tabular}{ccl}
$\mathrm{H}$ & $\frac{\Delta C}{\Delta \alpha}$ millimètres & \multicolumn{1}{c}{$\frac{1}{\mathrm{H}^{2}} \frac{\Delta \mathrm{C}}{\Delta \alpha}$} \\
562 & par degré & $0,186 \times 10^{-4}$ \\
1171 & 6,0 & 0,159 \\
2005 & 21,7 & 0,12 \\
3945 & 42 & 0,064 \\
7327 & 100 & 0,0096
\end{tabular}

Le couple exercé au minimum variait donc bien d'une manière voisine de la proportionnalité au carré du champ, dans les champs faibles.

Pour comparer la rapidité du phénomène dans cette nouvelle substance à celle de l'ancienne, j'exprime pour l'une et l'autre l'accroissemént du couple pour $1^{\circ}$ en pour cent de l'amplitude totale de la variation des couples :

$\begin{array}{ccc}\mathrm{H} & \text { Ancienne substance } & \text { Nouvelle substance } \\ 562 & \mathbf{5 , 4} 0 / 0 & 16,70 / 0 \\ 1171 & - & \mathbf{2 9 , 5} \\ 2005 & 9,3 & 34 \\ 3945 & 11,6 & 45 \\ 7327 & 6,9 & 23,7\end{array}$

Dans la nouvelle substance, le phénomène est environ quatre fois plus rapide que dans l'ancienne.

L'épaisseur un peu moindre du disque ne suffit pas à expliquer cette augmentation, qui aurait dû, tout au plus, réduire de moitié le champ démagnétisant dû à la forme. Le caractère accidentel du coefficient $n$, variable d'un échantillon à l'autre, me paraît être un argument, en faveur de l'hypothèse qui attribue la susceptibilité initiale de l'échantillon à sa structure grossière.

Puisque, dans l'ancienne substance, au minimum dans les champs faibles, l'aimantation tourne environ 80 fois plus vite que le champ (voir page 490̃), elle doit, dans la nouvelle, tourner environ 320 fois plus vite, si, ce qui est probable, $\mathrm{N}$ a à peu près la même valeur.

Une contre-épreuve consisterait à conserver la structure de l'échantillon, en faisant varier ses propriétés magnétiques. C'est précisément 
PROPRIÉTÉS MAGNÉTIQUES DE LA PYRRHOTINE 499 ce qui arrive au cours de l'étude thermique du magnétisme. Dans un travail fait en collaboration avec M. J. Kunz, qui paraîtra prochainement, nous publierons une série de courbes relevées à diverses températures représentant $\mathrm{C}$ en fonction de $\alpha$ pour une même valeur du champ. Les coefficients angulaires au minimum $\frac{\Delta C}{\Delta y}$ sont les mêmes pour toutes ces courbes. C'est bien ce qui doit arriver si la grandeur de $n$ est dépendante de la structure et non des propriétés magnétiques de la matière.

d) Au maximum, on déduit assez exactement des expériences le rapport des vitesses de rotation de l'aimantation et du champ. Il a pour expression

$$
\frac{\Delta \varphi}{\Delta \alpha}=\frac{\mathrm{H}}{\mathrm{H}+\mathrm{NI}_{\mathrm{M}}}
$$

et permet par conséquent de déterminer le champ démagnétisant maximum.

e) On relève aussi directement sur la courbe expérimentale donnant $C$ en fonction de $\alpha$ la valeur de $\frac{\Delta C}{\Delta \alpha}$ au maximum. Elle a pour expression :

$$
\frac{\Delta \mathrm{C}}{\Delta \alpha}=\mathrm{NI}_{\mathrm{M}}{ }^{2} \frac{\mathrm{H}}{\mathrm{H}+\mathrm{NI}_{\mathrm{M}}} .
$$

Je me suis servi à plusieurs reprises des formules (̋) et (6), qui sont commodes, pour des contrôles qui sont en partie équivalents à ceux que j'ai déjà indiqués et sur lesquels je n'insiste pas.

\section{CHAPITRE V.}

LE PLAN MAgNétiQue.

Dès le début des mesures sur la pyrrhotine, j'ai été frappé par la très grande différence d'intensité des propriétés magnétiques dans le plan de base du prisme hexagonal et perpendiculairement à ce plan. En tenant à la main un cristal que l'on approche d'un aimant, on se rend compte de l'attraction très vive qu'il éprouve et qui disparaît quand on le tourne de façon à présenter le plan de base et perpendiculairement aux lignes de force. 
Mesurant la grandeur de l'aimantation dans le plan magnétique d'une part $\left({ }^{1}\right)$, et la différence entre l'aimantation parallèle et perpendiculaire à ce plan de l'autre, j'ai trouvé ces quantités égales.

Ayant placé, dans le goniomètre magnétique de la page 474, une sphère de pyrrhotine, dont le plan magnétique était vertical, j'a déterminé pour tous les azimuts par rapport au champ la composante de l'aimantation parallèle au champ et la composante perpendiculaire au champ et horizontale. La $f g .16$ montre comment la recomposition de l'aimantation résultante, à partir de ces données expérimentales anciennes et relativement peu précises, donne des points situés tous sur la trace du plan magnétique dans le plan horizontal. Chaque direction de champ fournit deux points à cause de l'hystérèse.

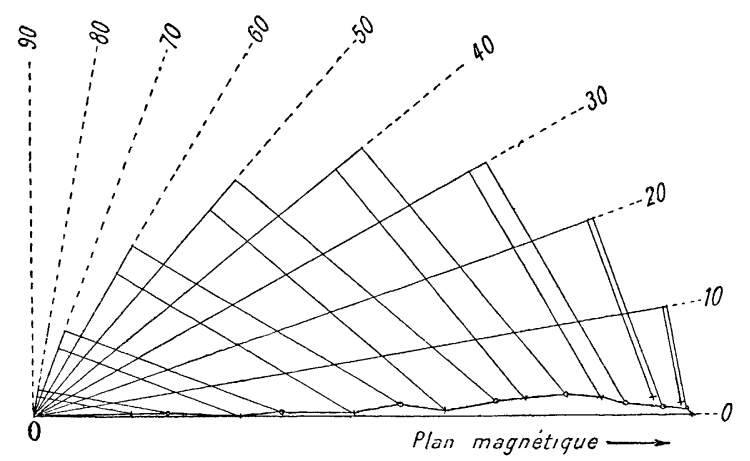

FIG. 16.

Le contrôle le plus effectif avait été obtenu en mesurant le courant induit dans une bobine placée dans le champ d'un électro-aimant, lorsqu'on y introduisait la pyrrhotine dont le plan magnétique avait été réglé perpendiculairement au champ.

Toutes ces expériences avaient été faites dans des champs relativement faibles de quelques centaines d'unités. J'ai tenté à plusieurs reprises de répéter les dernières dans des champs intenses, et je cite ici quelques-uns des résultats obtenus :

(1) C. R., t. CXXVI, p. 1099; 1898. - J. de Phys.. 3 série, t. VıII, p. $542 ; 1899$. 
Pyrrhotine normale (Morro Velho)

\begin{tabular}{|c|c|c|c|c|c|}
\hline \multicolumn{2}{|c|}{$\begin{array}{c}\text { Aimantation } \\
\text { dans le plan magnétique }\end{array}$} & \multicolumn{2}{|c|}{$\begin{array}{l}\text { Aimantation } \\
\text { perpendiculaire au } \\
\text { plan magnétique }\end{array}$} & \multicolumn{2}{|c|}{$\begin{array}{l}\text { En pour cent } \\
\text { de l'aimantation dans le } \\
\text { plan magnétique }\end{array}$} \\
\hline 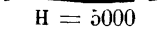 & $H=10000$ & $H=5000$ & $\mathrm{H}=10000$ & $H=5000$ & $H=10000$ \\
\hline 67,2 G. G. S & 74,7 & 4,76 & 7,23 & 7,1 & 9,7 \\
\hline 61,2 & 64,8 & 3,95 & 10,22 & 6,45 & 15,8 \\
\hline 65,1 & 74,1 & 5,95 & 9,74 & 9,1 & 13,2 \\
\hline 59,3 & - & 4,90 & - & & \\
\hline \multicolumn{6}{|c|}{ Pyrrhotine anormale (Bodenmais) } \\
\hline 54,6 & 57,8 & 7,52 & 11,47 & 13,8 & 19,8 \\
\hline
\end{tabular}

L'expérience donne donc une aimantation perpendiculaire au plan magnétique assez notable. Remarquons cependant que la plupart des causes d'erreurs, les erreurs de taille des disques, les erreurs d'orientation, la courbure quelquefois observée de l'édifice cristallin, les impuretés magnétiques consistant en matières étrangères ou en pyrrhotine de plan magnétique différemment orienté, tendent à faire apparaître cette aimantation. En rapprochant cette remarque de l'allure capricieuse des résultats d'expérience, on ne peut leur attribuer une portée décisive, et on doit recourir à une autre méthode.

Elle s'est présentée d'elle-même lorsque j'ai employé la méthode du couple exercé sur la substance à la détermination de la composante de l'aimantation parallèle au champ.

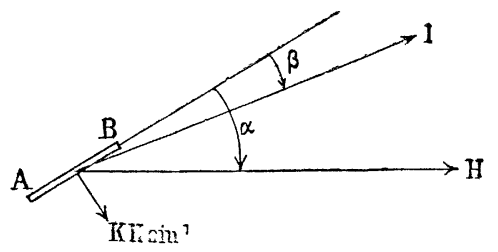

FIG. 17.

Suspendons un disque de pyrrhotine $\mathrm{AB}$ ( $f g .17)$ dans l'appareil à aimant tournant de la page 479, son plan magnétique placé verticalement. Soient I la composante horizontale de l'intensité d'aimantation, $\alpha$ l'angle du champ avec le plan magnétique et $\mathrm{C}$ le couple exercé sur la substance. On aura, si la propriété du champ magnétique est rigoureuse :

$$
\mathrm{C}=\mathrm{HI} \sin \alpha .
$$

J. de Phys., $4^{\circ}$ série, t. IV. (Juillet 190̈.) 
étant lu sur le cercle divisé de l'aimant, I est ainsi déterminé. Supposons maintenant que l'intensité d'aimantation s'écarte du plan magnétique d'un angle $\beta$, grâce à une faible susceptibilité $k^{\prime}$ normale à ce plan. Alors :

$$
\begin{gathered}
\sin \beta=\frac{k H \sin \alpha}{I} \\
G=H I \sin (\alpha-\beta)
\end{gathered}
$$

et, par suite, avec une grande approximation :

$$
\mathrm{G}=\mathrm{H} \sin \alpha(\mathrm{I}-k \mathrm{H} \cos \alpha) ;
$$

$\alpha$ n'a pas dépassé $10^{\circ}$. On peut donc, puisque $k \mathrm{H}$ est petit par rapport à $I$, écrire avec une approximation encore suffisante :

$$
\mathrm{C}=\mathrm{H}(\mathrm{I}-\mathrm{KH}) \sin \alpha .
$$

On mesurera donc, à la place de l'intensité d'aimantation réelle, une intensité d'aimantation apparente d'autant plus faible que le champ est plus intense.

Si les champs employés sont assez intenses pour produire la saturation, on observera par cette méthode ou une aimantation constante, ou une aimantation apparente décroissante, suivant que la susceptibilité normale sera ou ne sera pas nulle.

Plusieurs séries d'expériences m'ont parı confirmer d'abord la rigueur du plan magnétique. J'ai observé, par exemple, sur une substance normale de MorroVelho :

$\begin{array}{cc}\text { H } & \text { I (unité arbitraire) } \\ 1986 & 689 \\ 3930 & 732 \\ 7300 & 763 \\ 91500 & 768 \\ 10195 & 767 \\ 11040 & 765\end{array}$

Il est difficile d'atteindre la saturation dans un échantillon complexe formé de cristaux associés. On se place dans les conditions les plus favorables en dirigeant la plus grande composante parallèlement au champ. Mais l'étude des propriétés dans le plan magnétique a montré que l'obliquité de l'aimantation des plus petites composantes est alors loin d'être négligeable. 
PROPRIÉTÉS MAGNÉTIQUES DE LA PIRRHOTINE כ03

Ce que l'on observe est une aimantation apparente qui décroit avec le champ à cause de la susceptibilité normale et croît par suite de la saturation incomplète, et les deux effets peuvent se masquer mutuellement. Il est donc nécessaire de faire la correction des substances parasites par l'étude préalable du plan magnétique. Le résultat de ces opérations sur la substance 3 , à laquelle se rapportent la plupart des mesures de ce travail, est contenu dans le tableau suivant et représenté dans la fig. 15ั, page 493.

$\begin{array}{rcccc}\text { H } & \begin{array}{c}\text { Aimantation } \\ \text { apparente brute }\end{array} & \begin{array}{c}\text { Aimantation } \\ \text { apparente de la } \\ \text { matière simple }\end{array} & \begin{array}{c}\text { Correction par } \\ =314 \times 10^{-6}\end{array} & \begin{array}{c}\text { Aimantation } \\ \text { corrigée }\end{array} \\ 1992 & 43,2 & 43,9 & 0,6 & 46,5 \\ 4000 & 44,0 & 45,6 & 1,25 & 46,85 \\ 7310 & 44,3 & 44,9 & 2,2 & 47,2 \\ 10273 & 43,6 & 43,7 & 3,2 & 46,9 \\ 11140 & 43,4 & 43,6 & 3,5 & 47,1\end{array}$

L'aimantation apparente brute possède un maximum; l'aimantation apparente de la matière simple, représentée par la courbe Il, décroît régulièrement. La courbe $I$ et la dernière colonne du tableau se rapportent à cette aimantation corrigée, par addition d'une quantité égale à $314 \times 10^{-6} \mathrm{H}$ et rendue ainsi sensiblement constante. On doit donc admettre que la susceptibilité normale au plan magnétique est égale à

$$
k=314 \times 10^{-6}
$$

Les champs normaux pour lesquels elle est observée sont compris entre 0 et 2000 gauss. Elle correspond à une aimantation de 0,67 0/0 de la saturation, pour 1000 gauss. Si elle restait constante dans les champs plus élevés, elle conduirait à la saturation pour un champ de 150000 gauss. Elle rend suffisamment compte, avec les causes d'erreur invoquées, des résultats des expériences d'induction (p. 501).

Dans les premières expériences sur le plan magnétique(1), j’avais estimé que, pour une pyrrhotine de Morro Velho, l'aimantation perpendiculaire au plan magnétique ne dépassait pas $\frac{1}{680}$ de l'aimantation dans ce plan. Cette évaluation reposait sur l'opinion que, dans le

(1) C. R., t. GXXVI, p. 1099, $1998 ;-J$. de Plıys., 3e série, t. VIII, p. $542 ; 1899$. 
plan magnétique, la pyrrhotine pouvait être traitée, dans le cas de la sphère, comme une substance de susceptibilité notable, alors qu'en réalité elle était voisine de la saturation. La limite trouvée doit donc être portée à $\frac{3}{680}$. La détermination actuelle montre qu'elle n'est dépassée que pour des champs supérieurs à $6 \check{0} 0$ unités.

Il est intéressant de comparer la susceptibilité du fer dans les composés faiblement magnétiques ou paramagnétiques à cette susceptibilité normale.

Si l'on attribue le magnétisme de la pyrrhotine uniquement au fer qu'elle contient, on obtiendra la susceptibilité spécifique $\%$ du fer dans la pyrrhotine en divisant la susceptibilité par la densité du fer dans la pyrrhotine, qui est de 1,76.

On trouve :

$$
\%=175 \times 10^{-6},
$$

et pour la susceptibilité atomique :

$$
a \%=56 \%=0,0098 \text {. }
$$

Pour 9 composés ferreux et ferriques, la susceptibilité moléculaire $\% \cdot m$ est, par atome de fer dans la molécule, comprise entre 0,0127 et $0,0151\left(^{1}\right)$. Elle est égale à la susceptibilité atomique si l'on attribue les propriétés magnétiques uniquement au fer dans la molécule.

Rapprochons encore de ces données le paramagnétisme du fer et de la magnétite au-dessus de la température de pertedu ferromagnétisme, déterminés par M. Curie. M. du Bois (loc. cit., p. 486) définit la constante de Curie $\mathrm{C}$ par $\chi=\mathrm{C} \times(\Theta)$, où $\Theta$ est la température absolue. La constante de Curie atomique pour le fer aux hautes températures est :

$$
\mathrm{C} \times a=2,16 \text {, }
$$

et pour la magnétite, la constante de Curie moléculaire de la molécule $\mathrm{Fe}^{3} \mathrm{O}^{4}$ :

$$
\mathrm{G} \times m=3 \times 2,17 \text {. }
$$

On déduit de ces deux valeurs remarquablement concordantes $\left({ }^{2}\right)$, par extrapolation, une susceptibilité paramagnétique du feraux tem-

(') Du BoIs, Rapporls du Congrès int. de Phys., t. II, p. 496; 1900.

(2) Du Bors, loc. cit., p. 494. 
pératures ordinaires :

$$
\frac{\mathrm{C} \times a}{293}=\chi \cdot a=0,0074 \text {. }
$$

La susceptibilité atomique du fer dans la pyrrhotine, prise normalement au plan magnétique, est donc très voisine de la susceptibilité atomique du fer dans les corps paramagnétiques.

Il convient de lever une objection. Cette susceptibilité n'a été reconnue que grâce à son anisotropie. Une susceptibilité paramagnétique isotrope, superposée aux phénomènes déjà considérés, eût passé inaperçue et doit ètre recherchée.

Les objections qui rendent suspecte la méthode d'induction pour l'expérience de zéro ne s'appliquent pas au même titre à l'expérience analogue pour la mesure de l'intensité d'aimantation à saturation dans le plan magnétique. J'ai trouvé ainsi, en unités arbitraires, pour la même substance:

$\begin{array}{ccc}\mathrm{H} & \text { Aimantation brute } & \begin{array}{c}\text { Aimantation } \\ \text { de la matière simple }\end{array} \\ 1450 & \mathbf{4 4 , 7 5} & 38,4 \\ 2945 & 45,72 & 38,4 \\ 5125 & 46,78 & 38,4 \\ 5970 & 47,15 & 38,5 \\ 6980 & 47,46 & 38,4\end{array}$

L'aimantation est donc parfaitement constante. La saturation exclut donc une susceptibilité paramagnétique de même direction qu'elle, du même ordre degrandeur que celle des composés faiblement magnétiques du fer.

Si l'on craignait d'attribuer une trop grande importance à la coïncidence numérique des susceptibilités paramagnétiques reposant sur l'étude, assez exacte il est vrai, d'une seule substance, la dernière colonne de ce tableau suffirait à montrer que le ferromagnétisme et le paramagnétisme dépendent de la même cause profonde.

Remarquons enfin que, si l'on ne disposait que des champs inférieurs à 70 gauss, le phénomène serait linéaire, l'aimantation ne s'écartant de la ligne de facile aimantation que d'un centième tout au plus dans le plan magnétique et d'un deux-millième perpendiculairement à ce plan.

La méthode qui résulte de cette analyse des propriétés du plan magnétique a été employée à plusieurs reprises pour la détermi- 
nation de la composante de l'aimantation parallèle au champ en fonetion de l'azimut du cham $\rho$ dans le plan magnétique. Ainsi, les courbes de la $f i g .11$ et les courbes de la fig. 10 qui en dérivent ont été obtenues de cette manière. A cet effet, l'appareil représenté fig. 18 était suspendu au ressort de torsion, le petit disque $d$, gradué sur son bord conique, placé au centre du champ. Le disque de pyrrhotine, taillé parallèlement au plan magnétique, était collé sur ce petit
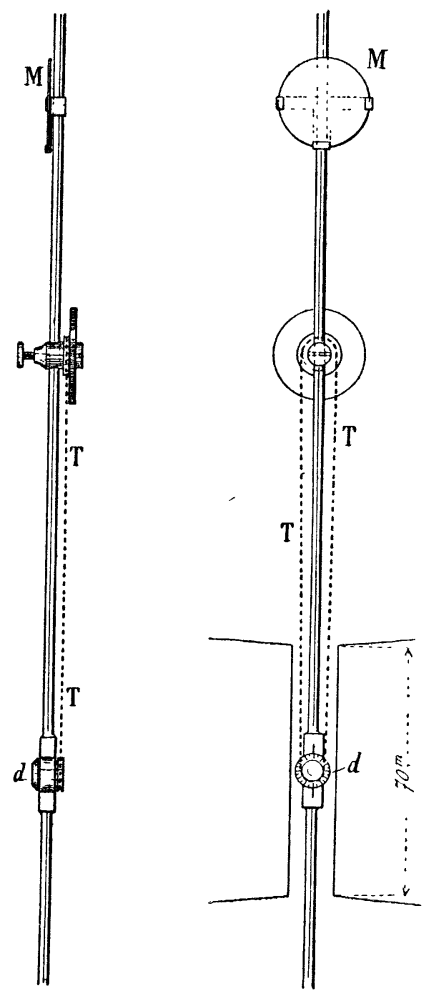

FIG. 18.

disque, que l'on pouvait faire tourner autour de son axe au moyen de la petite transmission $T$, sans le retirer du champ, tandis que l'on lisait sa graduation avec une loupe. L'appareil avait un frottement suffisant pour que le disque, réglé à un azimut déterminé, restât en place pendant le cours d'une expérience, consistant à observer les déviations du système suspendu au moyen du miroir M, l'aimant oc- 
PROPRIÉTÉS MAGNÉTIQUES DE LA PYRRHOTINE 507 cupant successivement des positions écartées de $+10^{\circ}$ et $\mathrm{de}-10^{\circ}$ à partir du parallélisme du champ avec le plan magnétique. Le couple mesuré a pour expression HI sin $10^{\circ}$, où I est la composante cherchée. Plusieurs petites corrections sont nécessaires, notamment celle de la susceptibilité normale qui a été faite avec $k=314 \times 10^{-6}$.

Il est nécessaire, en outre, pour l'interprétation de cette méthode, de savoir si une composante du champ perpendiculaire au plan magnétique altère ou non les phénomènes dans ce plan. Le travail d'aimantation peut s'écrire, pour un cristal à trois dimensions :

$$
d \mathrm{~T}=d\left(\mathbf{H}_{x} \mathbf{I}_{x}+\mathbf{H}_{y} \mathbf{I}_{y}+\mathbf{H}_{z} \mathbf{I}_{z}\right)-\left(\mathrm{I}_{x} d \mathrm{H}_{x}+\mathrm{I}_{y} d \mathrm{H}_{y}+\mathbf{I}_{z} d \mathbf{H}_{z}\right) .
$$

Soit XOY le plan magnétique, la condition $d \mathrm{~T}$ différentielle exacte donne :

$$
\frac{\partial \mathrm{I}_{x}}{\partial \mathrm{H}_{z}}=\frac{\partial \mathrm{I}_{z}}{\partial \mathrm{H}_{x}}, \quad \frac{\partial \mathrm{I}_{y}}{\partial \mathrm{H}_{z}}=\frac{\partial \mathrm{I}_{z}}{\partial \mathrm{H}_{x}} .
$$

Si $1_{z}$ est constamment nul, c'est-à-dire si le plan magnétique est rigoureux, les phénomènes dans ce plan ne dépendent donc pas d'une composante normale $\mathrm{H}_{z}$.

$\mathrm{Si}$, comme cela est possible, la susceptibilité normale $k=\frac{\mathrm{I}_{z}}{\mathrm{H}_{z}}$ est constante avec une approximation suffisante, l'on a encore :

$$
\frac{\partial \mathrm{I}_{x}}{\partial \mathrm{H}_{z}}=k \frac{\partial \mathrm{H}_{z}}{\partial \mathrm{H}_{x}}=0, \quad \frac{\partial \mathrm{I}_{y}}{\partial \mathrm{H}_{z}}=k \frac{\partial \mathrm{H}_{y}}{\partial \mathrm{H}_{z}}=0 .
$$

Je n'ai rencontré aucun fait qui fût en désaccord avec cette hypothèse. Notamment quand, dans la méthode exposée ci-dessus, j'ai répété l'expérience en donnant au champ un mouvement d'amplitude moitié moindre à partir du plan magnétique, les résultats ont été concordants.

Mais, pour élucider définitivement ce point, de nouvelles expériences seraient nécessuires, pour lesquelles il faudrait, sans doute, des moyens d'investigation plus puissants.

L'indépendance des propriétés dans le plan magnétique de toute composante normale permet de généraliser la méthode d'observation que nous venons de développer. Au lieu de donner au champ des oscillations de petite amplitude de part et d'autre du plan magnétique, on lui fait décrire, en s'arrêtant de $10^{\circ}$ en $10^{\circ}$, par 
כ08 ẆEISS. - PROPRIÉTÉS MAGNÉTIQUES DE LA PYRRHOTINE

exemple, un tour complet, et on lit les couples correspondant à ces diverses positions. La composante du champ dans le plan magnétique passe alors de la valeur maxima $\mathrm{H}$ à zéro, et de zéro à $-\mathrm{H}$, etc. On relève ainsi en une seule opération une courbe d'aimantation au sens habituel du mot. Le commencement OA de la courbe II $(f g .15)$, entre autres, a été déterminé de cette manière.

Détermination de l'aimantation de la pyrrhotine en valeur absolue.

Il suffit de déterminer en valeur absolue l'intensité à saturation pour pouvoir, par proportionnalité, y ramener les mesures relatives de toutes les intensités d'aimantation. L'intensité à saturation de l'échantillon qui a été l'objet des plus nombreuses expériences (substance 3) a été trouvée, par la méthode d'induction,

$$
\mathrm{I}_{\mathrm{M}}=47 \text { unités C. G. S. }
$$

Auparavant, l'aimantation de cette substance sans correction de l'obliquité des composantes parasites, dans un champ de 3800 gauss, avait été trouvée égale à $44,8 \mathrm{C}$. G. S. avec des appareils et une méthode opératoire différents.

Pour trois autres échantillons de Morro Velho que j'ai mesurés, j'ai trouvé des intensités supérieures :

$$
63,6, \quad 65,5, \quad 72,8 \text {. }
$$

M. J. Kunz, de son côté, a trouvé pour des pyrrhotines de même origine des résultats compris entre 64,2 et 66,7, par la méthode des couples, en déterminant en valeur absolue la constante du ressort de torsion.

L'intensité à saturation de la pyrrhotine de Morro Velho, tout en étant toujours du même ordre de grandeur, ne semble donc pas être invariable d'un échantillon à l'autre.

L'intensité à saturation des pyrrhotines anormales est extrêmement variable, elle est comprise, pour les échantillons qui ont été l'objet de déterminations absolues, entre 18,3 et 128.

(A suivre.) 